\title{
News Aggregators and Competition Among Newspapers on the Internet*
}

\author{
Doh-Shin Jeon ${ }^{\dagger}$ and Nikrooz Nasr ${ }^{\ddagger}$
}

July 16, 2014

\begin{abstract}
This paper considers how news aggregators affect the quality choices of newspapers competing on the Internet. To provide a microfoundation for the role of the aggregator, we build a model of multiple issues where newspapers choose their quality on each issue. The model captures both "business-stealing" and "readership-expansion" effects of the aggregator. We find that the aggregator leads newspapers to specialize their news coverage, and changes quality choices from strategic substitutes to strategic complements. Overall, the aggregator tends to increase the quality of newspapers and social welfare, but affects newspapers' profits in an ambiguous manner.
\end{abstract}

JEL Classification: D21, D43, L13, L82

Key words: Newspapers, News Aggregator, Internet, Quality, Strategic Substitutes, Strategic Complements, Advertising, Business-stealing, Readership-expansion, Opting Out.

\footnotetext{
${ }^{*}$ We thank Simon Anderson, Michael Baye, Gary Biglaiser, Bernard Caillaud, Dong Ook Choi, Joaquin Coleff, Kenneth Corts, Chrysanthos Dellarocas, Romain De Nijs, Lisa M. George, David Henriques, Bruno Jullien, ByungCheol Kim, Andras Niedermayer, Martin Peitz, Jeffrey Prince, Patrick Rey, Yossi Spiegel, and Alexander Wolitzky for useful comments and the participants of our presentation at the Conference on The Economics of Intellectual Property, Software and the Internet (Toulouse, 2013), Conference on "The Economics of the Postal Sector in the Digital World" (Toulouse, 2012), EARIE (2012), ICT workshop (Mannheim, 2012), ICT workshop (Porto, 2012), IIOC (2012), KISDI, Media Economics workshop (Bogota, 2012), Net Institute conference (Berkeley, 2013), PSE, SAET (2013), Searle Conference on Internet Search and Innovation (Northwestern, 2012), Sogang University and Sungkyunkwan university. We thank the NET Institute, www.NETinst.org, for financial support.

${ }^{\dagger}$ Toulouse School of Economics (GREMAQ, IDEI) and CEPR. dohshin.jeon@gmail.com

${ }^{\ddagger}$ Toulouse School of Economics. me@nikrooz.com
} 


\section{Introduction}

Newspapers are in stiff competition with new online media. Online media is the only media form which has experienced audience growth in the past decade, as can been seen from Figure 1. Among online media sources, news aggregators are the most important. According to Outsell report (2009), 57 percent of news media users now go to digital sources, and they are also more likely to turn to an aggregator (31 percent) than to a newspaper site ( 8 percent) or other news site (18 percent). Indeed, Pew Research Center (2012) shows that aggregators (Yahoo! News, Google News, MSN, AOL News and Huffington Post) attract more than half of the online news traffic in the US. In South Korea, $85 \%$ of the total traffic to newspaper sites originated from the news aggregators of top two domestic search engines; NAVER, the number one search engine, accounts for $70 \%$ alone. $^{1}$

The success of news aggregators has generated a heated debate about the effects of news aggregators on newspapers. At the heart of the debate is the effect on newspapers' incentives to produce high-quality content. The debate has already attracted the attention of governments and regulatory bodies. During 2009 to 2010, the FTC hosted three workshops on the Future of Journalism and has published a controversial "discussion draft" that hints at copyright reform and the protection of newspapers from aggregators. In Europe, the German government recently adopted a project to introduce "Lex Google," a law intended to make Google pay for indexing the content of German news sites. ${ }^{2}$ A similar law was proposed in Italy $^{3}$ and French newspapers wanted the same. ${ }^{4}$ Recently, France President François Hollande revealed the settlement that Google would create a $€ 60$ million fund to help the French newspapers develop their Internet presence. ${ }^{5}$

In the debate on news aggregators, on the one hand, content producers argue that news aggregators make money by stealing high-quality content. Since this money is pulled out of content producers' pockets, they have less incentive to produce high-quality content. For instance, according to Rupert Murdoch (2009), chairman of News Corp.:

When this work is misappropriated without regard to the investment made, it destroys the economics of producing high-quality content. The truth is that the 'aggregators' need news organizations. Without content to transmit, all our flat-

\footnotetext{
${ }^{1}$ See http://www.rankey.com/blog/blog.php?type=column\&sub_type $=$ all \&writer $=\&$ no $=327 \&$ page $=9 \&$ search_type $=$ subject\&search_wd=

2"Polémique sur la 'Lex Google' en Allemagne," Le Monde, 30 Aug. 2012

3"Taxing times," The Economist, 10 Nov. 2012

${ }^{4}$ Le Figaro, les Echos and le Nouvel Observateur are in favor of Google tax:

"Taxe Google : Le Figaro, les Echos et le Nouvel Obs veulent être payés," ZDNet, 11 Sep. 2012

5"Google Settles Dispute with French Newspapers," Wall Street Journal, 1 Feb. 2013
} 


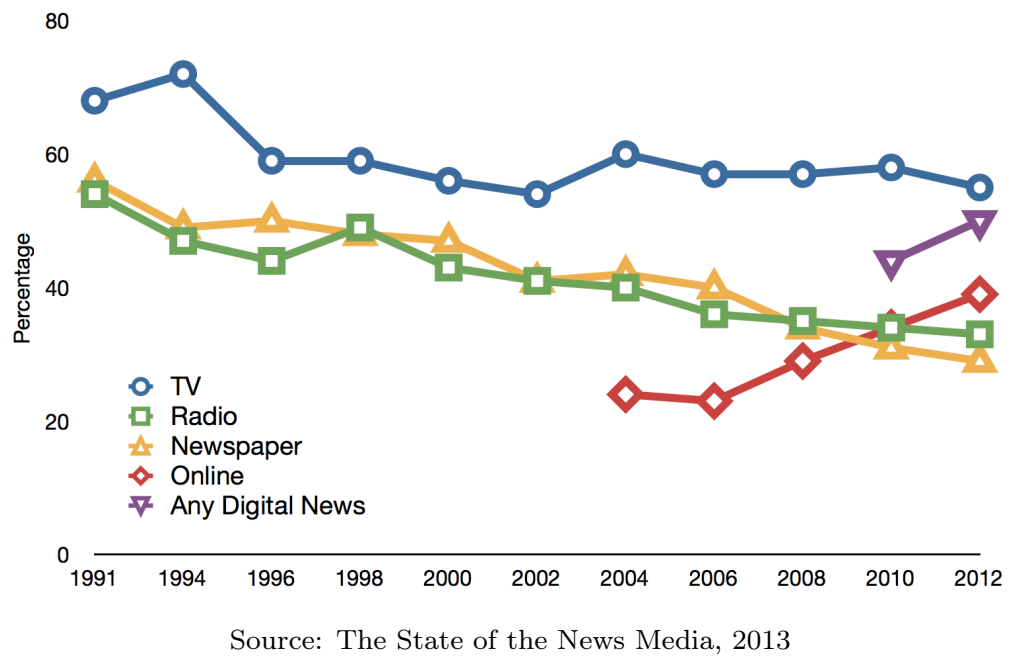

Figure 1: Where People Got News Yesterday

screen TVs, computers, cell phones, iPhones and blackberries, would be blank slates.

(p.13).

On the other hand, news aggregators argue that aggregation drives profitable traffic to the news sites themselves. In a response to the FTC report (2010), Google (2010) claimed to send more than four billion clicks per month to news publishers via Google Search, Google News, and other products. Google's claim is that each click - each visit - provides publishers with an opportunity to show ads, register users, charge for access to content, and so forth.

In this paper, we study how the presence of a news aggregator affects competition between newspapers on the Internet and their quality choice. In our model, we allow each newspaper to choose quality for each separate issue. Hence, each newspaper's strategy has both a vertical dimension (through quality choice) and a horizontal dimension (through choice of issues to cover with high quality). Our multi-issue model provides a microfoundation for the role of the aggregator. We embed this multiple-issue feature into the classic Hotelling model where product differentiation can be interpreted as ideological differentiation as in Gabszewicz, Laussel, and Sonnac (2001) and Mullainathan and Shleifer (2005).

We have in mind a sequential reading process in which a reader first reads a homepage (i.e., an index page) and then click on the issues that she wants to read more about. More precisely, a reader spends a mass one of attention on the homepage by reading titles and abstracts, and spends additional $\delta>0$ units of attention per article by clicking through to the original article if it has high-quality content. We model the aggregator to resemble Google News: it has no original articles and its homepage provides a link to the highest quality article on each issue. Therefore, 
when a reader switches from her preferred newspaper to the aggregator, she benefits from better quality articles but suffers from worse preference (ideological) match. This microfoundation allows to capture two opposite effects at the core of the debate on news aggregators: the businessstealing effect and the readership-expansion effect. The former occurs in terms of homepage consumption as long as some readers switch from a newspaper to the aggregator. The latter arises at each article level: since the aggregator improves the match between readers' attention and high-quality content, it expands the readership for high-quality articles even if the total mass of readers is assumed to be constant. ${ }^{6}$

In the baseline model, we consider two symmetric newspapers. We find that the presence of an aggregator would lead each newspaper to specialize in a different set of issues (i.e., maximum differentiation) when advertising revenue increases substantially with increase in quality (i.e., $\delta$ high) and would lead both newspapers to invest in the same issues (i.e., minimum differentiation) otherwise. When both newspapers use the maximum differentiation strategy, the presence of the aggregator changes the strategic interactions of quality choices from strategic substitutes to strategic complements. As a consequence, the aggregator increases the average quality of newspapers, which in turn increases consumer surplus and social welfare. However, the effect on the newspapers' profits is ambiguous.

The intuition for the change in the strategic interactions is the following. Without the aggregator, if newspaper 2 chooses a higher quality, this decreases the market share of newspaper 1 and hence reduces 1's marginal advertising revenue from increase in quality. On the contrary, when both newspapers use the maximum differentiation strategy in the presence of the aggregator, if newspaper 2 increases its quality, surprisingly, this expands the market share of the aggregator. This in turn implies that the high-quality content of newspaper 1 can reach a larger number of readers. Therefore, 2's quality increase raises 1's marginal advertising revenue from quality increase.

When the aggregator induces minimum differentiation, it has zero market share and we find that there is a continuum of symmetric equilibria. However, when we allow each newspaper to choose to opt out (i.e., to break the hyperlink to the aggregator's site), only the equilibrium quality without the aggregator survives. Therefore, the introduction of an opt-out option leads to a sharp prediction: the aggregator leads either to no change or to the maximum differentiation equilibrium.

Section 6 studies various extensions to make the model closer to the real world and to show the robustness of our main results: asymmetric issues, imperfect certification technology, third-

\footnotetext{
${ }^{6}$ In reality, many news aggregators are run by search engines and facilitate news consumption of search engine users. This suggests that news aggregators increase the total mass of readers. Since we shut down this channel by assuming that the mass of readers is constant, the readership-expansion effect we capture is a lower bound.
} 
party content, paywall. In particular, the extension involving third-party content in Section 6.3 allows for asymmetry among newspapers. In reality, there are many small news sites which would receive negligible traffic without aggregator. Therefore, these sites have strong incentives to use "the maximum differentiation and opt-in strategy" to attract traffic from the aggregator. In order to capture this scenario, we assume that by using the aggregator, consumers can get additional utility, $u_{T}$, where $T$ represents third-party content. When $u_{T}$ is important enough, we find that it is a dominant strategy for each newspaper to adopt the maximum differentiation and that quality choices are strategic complements. We find a unique symmetric equilibrium in which quality increases with $\delta$. To obtain a lower bound on $\delta$, we rely on empirical findings of Athey and Mobius (2012) and Chiou and Tucker (2012) (explained below) and find that the aggregator increases the quality of newspapers and that no newspaper has an incentive to opt out. In addition, Section 6.4 shows that our main results are robust to allowing for paywalls if competition among newspapers is fierce enough.

The rest of the paper is organized as follows. After reviewing the related literature in Section 1.1, we present the model in Section 2. In Section 3, we study newspaper competition without aggregator as a benchmark. Section 4 studies how an aggregator affects newspaper competition. Section 5 compares the outcome without the aggregator to the one with the aggregator in terms of quality, consumer surplus, profit and social welfare. Section 6 provides various extensions. Section 7 concludes. All the proofs except for the short proof of Lemma 2 are gathered in the Appendix A or in the Supplementary materials.

\subsection{Literature review}

The closest papers to ours among theoretical papers on news aggregators are Dellarocas, Katona, and Rand (2012) and Rutt (2011). Dellarocas, Katona, and Rand (2012) consider a single-issue model with focus on interactions between quality choice and link decisions (i.e., every newspaper can provide a link to a rival's content). The aggregator benefits consumers by providing links to the highest quality content. They show that the presence of an aggregator might decrease (increase) competition among content providers if content providers can (can not) link to each other. Rutt (2011) uses an all-pay auction model to study newspapers' choice of quality and price when there are two types of consumers (loyal ones and searchers). A loyal consumer reads only her preferred newspaper while a searcher uses an aggregator to read the highest quality one among free newspapers. He finds that as the fraction of searchers increases, free newspapers choose higher quality while the rest choose lower quality. The major difference between our paper and these is that we consider a model of multiple issues with endogenous quality and coverage. This together with sequential reading process (from homepage to articles) allows us to 
provide a microfoundation for the role of the aggregator and capture the business-stealing and readership-expansion effects. Furthermore, our result that the aggregator changes the strategic interactions of quality choices from strategic substitutes to strategic complements does not exist in these previous papers. ${ }^{7}$

There are three empirical papers on news aggregators (Athey and Mobius, 2012, and Chiou and Tucker, 2012). These papers provide evidence for the dominance of the readership-expansion effect over the business-stealing effect. Chiou and Tucker (2012) study a natural experiment where Google News had a dispute with the Associated Press and hence did not show Associated Press content for some period. They find that after the removal of Associated Press content, users of Google News subsequently visited other news sites less often than users of Yahoo! News, which did not remove Associated Press content. They conclude that users of aggregators are more likely to seek additional sources and read further rather than being satisfied with the summary. Athey and Mobius (2012) study a case where Google News added local content to its homepage for those users who chose to enter their location. By comparing the consumers who use this feature with controlled users, they find that users who adopted the feature increased their usage of Google News, which in turn led to additional consumption of local news. They conclude that their results support the view that news aggregators are complementary to local news outlets. George and Hogendorn (2013) use a major redesign of Google News on June 30, 2010 placing a permanent strip of geo-targeted local news headlines and links onto the Google News front page and find that adding geo-targeted links increases both the level and share of local news consumed online.

More generally, since we model the aggregator as a multilateral platform for interconnection that provides certification service, our work builds a connection between two important literatures in industrial organization: the one on interconnection and/or compatibility (Farrell and Saloner, 1985, 1986, Katz and Shapiro, 1985, Crémer, Rey, and Tirole, 2000 etc.) and the one on information intermediary (Biglaiser, 1993, Lizzeri, 1999, Baye and Morgan, 2001 ). In particular, Jeon and Menicucci (2011) study interconnection among academic journal websites, either through a multilateral platform (such as CrossRef) or through bilateral arrangements. CrossRef is similar to news aggregator in the sense that it allows a reader to seamlessly move from one article to another by clicking on a link. However, the news aggregator in our model performs quality certification while this function is absent in CrossRef. ${ }^{8}$

\footnotetext{
${ }^{7}$ Calzada and Ordóñez (2012) study a newspaper's reaction to the aggregator in terms of versioning (and linking) decisions in the framework of a monopolist's second-degree price discrimination. George and Hogendorn (2012) consider a model of two-sided market in which news aggregators increase multi-homing viewers. They find that the switching of a given mass of viewers from single-homing to multi-homing is likely to reduce (increase) a news outlet's advertising revenue if the outlet initially has a high (small) share of exclusive viewers.

${ }^{8}$ In addition, our paper differs from Jeon and Menicucci (2011) in terms of the strategic variables considered:
} 
Our paper also builds on the literature on two-sided markets (Caillaud and Jullien, 2001, 2003, Rochet and Tirole, 2003, 2006, Anderson and Coate, 2005, Armstrong, 2006, Hagiu, 2006, Weyl, 2010). Two-sided markets can be roughly defined as industries where platforms provide intermediation services between two (or several) kinds of users. Typical examples include dating agencies, payment cards (Rochet and Tirole, 2002), media, operating systems (Parker and Van Alstyne, 2005), video games (Hagiu, 2006) and academic journals (Rochet and Jeon, 2010). In such industries, it is vital for platforms to find a price structure that attracts sufficient numbers of users on each side of the market. In the application to media (Gabszewicz, Laussel, and Sonnac, 2001, Anderson and Coate, 2005, Armstrong, 2006, Peitz and Valletti, 2008, Crampes, Haritchabalet, and Jullien, 2009), the two sides refer to readers and advertisers. Since we model the aggregator as a technology and study how the technology affects media competition, our paper is related to Anderson and Gans (2011) and Athey, Calvano, and Gans (2012). ${ }^{9}$ Instead of explicitly modeling competition in the market for advertising, we describe this market with a reduced-form (like Gabszewicz, Laussel, and Sonnac, 2001) ${ }^{10}$ in order to focus on rich strategic interactions in the newspaper content market. In addition, with this approach, we intend to capture the fact that newspapers compete with other media and non-media firms such as Craigslist in the advertising market.

\section{Model}

We consider two newspapers and one aggregator and study their competition on the Internet. To provide a microfoundation for the role of the aggregator, we introduce into the classic Hotelling model (Hotelling, 1929, Tirole, 1988, p. 279) some novel features - multiple issues and endogenous choice of quality and coverage - as is explained below.

\footnotetext{
we study how a news aggregator affects newspapers' choice of content (when content is free) whereas Jeon and Menicucci (2011) study how interconnections interact with pricing of academic journals for given content.

${ }^{9}$ Anderson and Gans (2011) study content providers' reaction to ad avoidance technologies and find that their adoption increases advertising clutter and may reduce total welfare and content quality. Athey, Calvano, and Gans (2012) study how applying consumer tracking technology to advertising affects competition between online news media.

${ }^{10}$ As in Gabszewicz, Laussel, and Sonnac, 2001, we can add a last stage after readers made choices among media outlets. Since each newspaper has monopoly power to sell access to its readers, it can charge a monopoly price to advertisers. Our assumption is that this monopoly price is proportional to the total attention of readers.
} 


\subsection{Newspapers and Consumers}

Throughout the paper, we assume that consumers single-home, which means that without the aggregator, a consumer consumes only one of the two newspapers. ${ }^{11}$ In the presence of the aggregator, a consumer chooses one among newspaper 1, newspaper 2 and the aggregator.

\subsubsection{Product Differentiation}

The two newspapers are located at the extreme points of a line of length $1:^{12}$ newspaper 1 on the left extreme point and newspaper 2 on the right extreme point. Mass 1 of consumers are uniformly distributed on the line. A location in the line represents the ideological view of a consumer or a newspaper (Mullainathan and Shleifer, 2005, and Gentzkow and Shapiro, 2011). If a consumer located at $x$ consumes an article from a newspaper located at $y$, the consumer incurs a transportation cost of $t|x-y|$ with $t>0$. The transportation cost represents utility losses due to imperfect preference matching. At long-term, the presence of news aggregators might affect ideological positions of newspapers. For our analysis, we take a mid-term perspective and assume that the ideological positions are given.

\subsubsection{Multiple Issues and Choice of Quality and Coverage}

There are multiple issues which each newspaper covers. For tractability, we assume that there is a continuum of issues. Let $S$ be the set of issues. On each given issue, a newspaper can provide either high or low-quality content. Even if ideological position might compromise the quality, each newspaper still can choose different levels of quality for given ideological position. So the strategy of newspaper $i$, with $i \in\{1,2\}$, is a subset of issues $s_{i} \subset S$ which it covers with highquality content; for the remaining $S-s_{i}$ issues, the quality of content is low. We interpret issues broadly such that they include not only breaking events such as earthquake, airline disaster, election but also some broad topics of social concern such as climate change, income inequality, genetically modified organism etc. An article has high-quality if it is accurate, complete, timely, original, engaging etc. Writing a high-quality article typically requires in-depth investigation. On the contrary, a low-quality article is based on the information obtained from some intermediaries such as Associated Press.

\footnotetext{
${ }^{11}$ This assumption is made to capture the main technological difference between the aggregator and newspapers in that the former allows consumers to have access to content from all newspapers. Since we consider only two newspapers, the assumption is needed. However, if we consider a large number of newspapers, we can allow consumers to read two or three newspapers without using the aggregator and still capture the technological difference.

${ }^{12}$ We follow here the maximum differentiation result in the Hotelling model. Mullainathan and Shleifer (2005) rediscover the maximum differentiation result in the context of media bias. Our results would hold for any symmetric locations of the newspapers.
} 
Let $\mu(s)$ represent the measure of any set $s \subset S$. Without loss of generality, assume $\mu(S)=1$. Then, $\mu\left(s_{i}\right)$ represents the average quality of newspaper $i$. Therefore, the strategy $s_{i}$ has a vertical dimension in terms of average quality: from now on, we will refer to $\mu\left(s_{i}\right)$ as the quality of newspaper $i$. Furthermore, even when both newspapers choose the same quality, the strategy has a horizontal dimension: each newspaper can cover, with high-quality content, either an identical or a different subset of issues. Given $0<\mu\left(s_{1}\right), \mu\left(s_{2}\right) \leq 1 / 2$, for newspaper $i \in\{1,2\}$, if $i$ chooses $s_{i}$ such that $s_{i} \cap s_{j}=\emptyset$, we say that $i$ uses the maximum differentiation strategy (equivalently, the specialization strategy). If $i$ chooses $s_{i}$ such that $\mu\left(s_{1} \cap s_{2}\right)=\min \left(\mu\left(s_{1}\right), \mu\left(s_{2}\right)\right)$, then we say that $i$ uses the minimum differentiation strategy (equivalently, the no-specialization strategy).

\subsubsection{Consumer Preferences}

We have in mind a sequential reading process in which a reader first reads a homepage (i.e., an index page) and then clicks on the issues that she wants to read more about. A homepage provides the title, a summary and a link to the original article on each issue. After reading the homepage, each reader decides whether to click on the links to read the original articles. In the baseline model, we assume that all issues have the same probability of click and that this probability only depends on the quality of the article covering a given issue. If an article has high quality, the probability of clicking on its link is normalized at one; if an article has low quality, readers do not click on its link. ${ }^{13}$ A reader spends a mass one of attention on the homepage and spends $\delta>0$ additional units of attention at each original article only if it is of high-quality. ${ }^{14}$ In Section 6.1, we discuss the more realistic case in which some major issues have a higher probability of click than the other issues.

Let $u_{0}$ represent a consumer's utility net of attention cost from reading the homepage of a newspaper. By reading a homepage, a consumer has a rough understanding about the major events of the day, which gives her a large benefit. Therefore, we assume $u_{0}>t$, which implies that each consumer consumes one of the newspapers' homepages. It is a standard full participation assumption in the Hotelling model. In the case of asymmetric issues, $u_{0}$ can be interpreted as the utility from reading both homepage and high-quality articles on major events (see Section 6.1). Let $\Delta u>0$ represent the utility increase net of attention cost that a consumer experiences from clicking on the link and reading an original article of high-quality. Then, the utility that a consumer located at $x$ obtains from consuming newspaper 1 or 2 is given by

$$
U^{1}(x)=u_{0}+\mu\left(s_{1}\right) \Delta u-x t
$$

\footnotetext{
${ }^{13}$ Alternatively, after clicking on the link to a low-quality article, a reader immediately stops reading it.

${ }^{14}$ If it is of low-quality, a reader does not find it worthwhile to spend extra attention on it.
} 


$$
U^{2}(x)=u_{0}+\mu\left(s_{2}\right) \Delta u-(1-x) t .
$$

Define $\beta$ as $\beta \equiv \Delta u / t$. We can interpret $\beta$ as a measure of disloyalty, in the sense that the smaller $\beta$ is, the more loyal are consumers to their newspapers. To ensure that each newspaper has a positive market share in the presence of the aggregator, we assume: ${ }^{15}$

A1: $\beta<1$.

\subsubsection{Advertising Revenues and Content Production Technology}

We consider a business model based on advertising in which newspapers' Internet content is free. Each unit of attention brings an advertising revenue of $\varpi>0$. In Section 6.4, we allow each newspaper to charge a price and show that the main results are robust.

For tractability, we model the cost of investing in news quality by a quadratic function. Furthermore, we are interested in a situation in which the two strategic decisions are taken separately: the choice of (average) quality, on the one hand, and the choice of differentiation in terms of issues covered with high-quality - on the other hand. Therefore, we assume that the cost of investing in a subset $s_{i}$ of measure $\mu\left(s_{i}\right)$ for newspaper $i \in\{1,2\}$ is given by

A2:

$$
C\left(\mu\left(s_{i}\right)\right)= \begin{cases}\infty & \mu\left(s_{i}\right)>\frac{1}{2} \\ c \mu\left(s_{i}\right)^{2} & \mu\left(s_{i}\right) \leq \frac{1}{2}\end{cases}
$$

where $c>0$ is a positive constant. Given that writing high-quality articles requires in-depth investigation, the cost of covering all issues with high quality for a single newspaper must be prohibitive. A2 means that it is too costly for one newspaper to cover more than half of all issues with high quality. A milder assumption suffices in the more realistic case in which issues are heterogenous in terms of probability of click (see Section 6.1). Limiting $i$ 's choice to $\mu\left(s_{i}\right) \leq \frac{1}{2}$ also serves the purpose of allowing each newspaper to make the two decisions separately. Without this assumption, the two choices cannot be made independently: for instance, when $\mu\left(s_{1} \cup s_{2}\right)=1$, increasing $i$ 's quality implies an increase in $\mu\left(s_{1} \cap s_{2}\right)$. In general, when there is no upper bound on the quality of an article on an issue, each newspaper is able to make the two decisions separately. We introduce the restriction $\mu\left(s_{i}\right) \leq \frac{1}{2}$ to capture this situation in our simple model with an exogenous upper bound on the quality of an article. ${ }^{16}$

\footnotetext{
${ }^{15}$ In the absence of the aggregator, it is sufficient to have $\beta<2$ to discard the cornering equilibrium.

${ }^{16} \mathrm{~A}$ model of continuous quality choice with no upper bound would be far less tractable without delivering much new insight. Even our simple model becomes technically involved because of the challenges arising from providing a microfoundation for the utility that a consumer obtains from the aggregator. As is shown in Lemma
} 
Thus, in the absence of the aggregator, the profit of newspaper $i \in\{1,2\}$ is

$$
\pi_{i}\left(s_{i}\right)=\varpi \alpha_{i}\left[1+\mu\left(s_{i}\right) \delta\right]-C\left(\mu\left(s_{i}\right)\right)
$$

where $\alpha_{i}$ is the market share of newspaper $i$.

In what follows, without loss of generality, we normalize $\varpi$ to one since what matters is only $c / \varpi$. However, the interpretation of our results will be done in terms of $c / \varpi$ (see the end of Section 5).

\subsection{Aggregator}

\subsubsection{Benefit and cost of using the aggregator}

The value-added of an aggregator consists in recognizing high-quality content ex post. In the real world, some aggregators, like Huffington Post, use editorial staff, while others, like Google News, use an algorithm to find high-quality content. After finding high-quality articles, each aggregator posts them on its site. This, however, can be done in different ways. Some, like Yahoo! News, post the whole article on their site, with no link to the original content. Usually, this is because the aggregator pays the newspaper for that content and hence has the right to publish it. In 2006, Yahoo! signed an agreement with Newspaper Consortium ${ }^{17}$ to use their content. Others, like Google News, show the title and a short summary and provide a link to the original article. The first pages and sample articles of Google News can be seen in Figures 7 and 8. These two types of aggregators bring revenue to newspapers in different ways: the first by buying a content license, and the second by sending traffic to newspaper sites.

We model an aggregator along the lines of Google News and relegate the licensing issue to future work. Hence, the aggregator in our model provides only a homepage without having its own original articles. It benefits consumers by improving the match between their attention and highquality content. More precisely, for each issue, the aggregator chooses one article and publishes its title and summary with a link to the original article. In the baseline model, we assume that the aggregator chooses the highest quality article for each issue and that if both newspapers produce the same quality articles on a given issue, it chooses one of them with an equal probability. Section 6.2 considers the more realistic case of imperfect certification technology and shows that the main results are robust to introducing this imperfection. Providing one link per issue is a

3 , in the presence of the aggregator, the denominator in the expression for a given newspaper's market share is a function of the strategies $\left(\mu\left(s_{1}\right), \mu\left(s_{2}\right)\right)$, which makes the analysis complex. This is why we consider a quadratic cost function.

${ }^{17}$ http://www.npconsortium.com/

"Is Yahoo a Better Friend to Newspapers Than Google?,"New York Times, 8 Apr. 2009 
realistic assumption. For instance, Google News provides one link per issue for all issues except for the top story, for which it shows multiple links (see Figure 8). ${ }^{18}$

A consumer who goes to the aggregator's homepage spends a mass one of attention on the homepage. In addition, the consumer clicks on the link of each high-quality article and spends $\delta$ units of attention (per article) on the newspaper site to which she is directed. The consumer does not click on the links to low-quality articles. ${ }^{19}$ Therefore, using the aggregator over her preferred newspaper allows a consumer to access more high-quality content, at a higher cost of preference mismatch. The business-stealing effect captures the reduction in the traffic to the homepages of the newspapers since some readers switch to the homepage of the aggregator. However, there is also a readership-expansion effect since high-quality articles of a given newspaper can reach not only its loyal readers, but also those using the aggregator. The latter includes consumers who would read the rival newspaper if there were no news aggregator.

\section{$2.3 \quad$ Timing}

In what follows, we analyze the following two-stage game.

- Stage 1: each newspaper $i$ simultaneously chooses $s_{i}$.

- Stage 2: if there is no aggregator, each consumer chooses one of the two newspapers (otherwise, one among the two newspapers and the aggregator).

\section{$3 \quad$ No Aggregator}

In this section, we analyze the two-stage game without the aggregator. Then, what matters is only $\mu\left(s_{i}\right)=\mu_{i}$ for $i=1,2$, because of our single-homing assumption. As usual we use backward induction and start from Stage 2 .

Let $x$ denote the location of the consumer who is indifferent between 1 and 2 , which is determined by:

$$
\mu_{1} \Delta u-t x=\mu_{2} \Delta u-t(1-x)
$$

Equivalently, we have

$$
x=\frac{1}{2}+\frac{\beta}{2}\left(\mu_{1}-\mu_{2}\right) .
$$

\footnotetext{
${ }^{18}$ In reality, Google News indexes content from 25000 news outlets and hence it does not make sense to assume that the aggregator provides links to all newspapers on every issue even if it seems plausible to provide two links per article in our simple model.

${ }^{19}$ Alternatively, she might click the link of a low-quality article but quickly stop reading the article upon realizing that the quality is low.
} 
From A1, we have $0<x<1$. Therefore, each newspaper's market share is positive: $0<\alpha_{i}<1$ for $i=1,2$.

Newspaper $i$ 's profit is given by

$$
\pi_{i}=\left[\frac{1}{2}+\frac{\beta}{2}\left(\mu_{i}-\mu_{j}\right)\right]\left[1+\mu_{i} \delta\right]-c \mu_{i}^{2} \text { for }\left(\mu_{i}, \mu_{j}\right) \in[0,1 / 2]^{2} .
$$

If $c \leq \beta \delta / 2$, the profit function is convex. As $\pi_{i}^{\prime}(0)=\beta+\delta-\beta \delta \mu_{j}>0$ for any $\mu_{j} \in[0,1 / 2]$, newspaper $i$ 's best response is $1 / 2$ for any $\mu_{j} \in[0,1 / 2]$. If $c>\beta \delta / 2$, the profit function is strictly concave. The best reply function of $i$ is given by

$$
B R_{i}^{N}\left(\mu_{j}\right)=\left\{\begin{array}{cl}
\frac{1}{2} & \text { if } \mu_{j} \leq 1-\frac{2 c-(\beta+\delta)}{\beta \delta}, \\
\frac{\beta+\delta-\beta \delta \mu_{j}}{4 c-2 \beta \delta} & \text { if } \mu_{j}>1-\frac{2 c-(\beta+\delta)}{\beta \delta} ;
\end{array}\right.
$$

where the superscript $N$ means 'no aggregator'. In this case, the slope of the best reply function is either zero or $-\beta \delta /(4 c-2 \beta \delta)$. Therefore, we can conclude:

Lemma 1. In the absence of the aggregator, newspapers' quality choices $\left(\mu_{1}, \mu_{2}\right)$ are strategic substitutes.

If newspaper $j$ increases its quality, this reduces newspaper $i$ 's market share and thereby $i$ 's marginal revenue from an increase in quality. This is why quality choices are strategic substitutes. $^{20}$ Figure 2 describes newspaper 1's best reply function when $c>\beta \delta / 2$.

Let $\left(\mu_{1}^{*}, \mu_{2}^{*}\right)$ denote the equilibrium quality in the absence of the aggregator. The next proposition shows that there is a unique equilibrium.

Proposition 1. Under A1 and A2, in the absence of the aggregator, there is a unique equilibrium, which is symmetric. In the equilibrium,

(i) the average quality of each newspaper is

$$
\begin{array}{rlrlrl}
\mu^{*} & =\mu_{1}^{*}=\mu_{2}^{*}=\frac{1}{2} & \text { if } & 0 \leq c \leq \frac{\delta \beta}{4}+\frac{\delta}{2}+\frac{\beta}{2}, \\
\mu^{*} & =\mu_{1}^{*}=\mu_{2}^{*}=\frac{\delta+\beta}{4 c-\delta \beta} & \text { if } & c & >\frac{\delta \beta}{4}+\frac{\delta}{2}+\frac{\beta}{2} ;
\end{array}
$$

(ii) the profit of each newspaper is $\pi^{*}=-c \mu^{*^{2}}+\frac{\delta}{2} \mu^{*}+\frac{1}{2}$.

One can easily check that $\mu^{*}$ and $\pi^{*}$ are increasing in $\delta$ and decreasing in $c . \mu^{*}$ is increasing in $\beta$ but $\pi^{*}$ is decreasing in $\beta$. This implies that newspapers benefit from customer loyalty but that their quality decreases with loyalty.

\footnotetext{
${ }^{20}$ The reasoning behind this is similar to that of quantities being strategic substitutes in Cournot competition: an increase in firm $j$ 's quantity reduces the price of firm $i$ 's good and hence the latter's marginal revenue from production. This intuition still holds if we allow newspapers to charge for subscriptions: for any given prices, quality choices are strategic substitutes.
} 


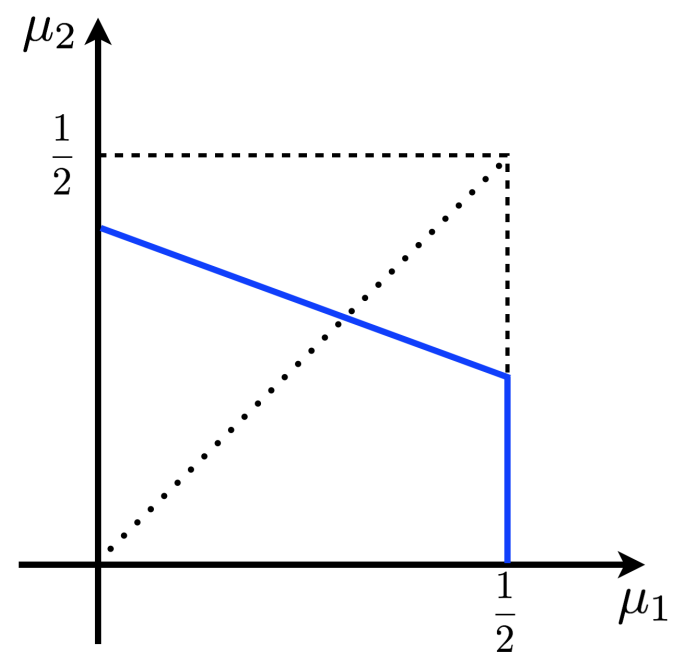

Figure 2: Best reply function of newspaper 1 when there is no aggregator

From now on, we assume that the equilibrium quality without the aggregator is interior (i.e., $\left.\mu^{*} \in(0,1 / 2)\right):$

A3: $c>\frac{\delta \beta}{4}+\frac{\delta}{2}+\frac{\beta}{2}$.

If A3 does not hold, each newspaper $i$ 's best reply is $\mu_{i}=\frac{1}{2}$ for any $\mu_{j} \in[0,1 / 2]$, which is not interesting.

\section{Aggregator}

In this section, the two newspapers compete in the presence of an aggregator.

\subsection{Market shares for given qualities}

Given $\left(s_{1}, s_{2}\right)$, the utility that a consumer with location $x$ obtains from using the aggregator is given by:

$$
\begin{aligned}
U^{A g g}(x) & =u_{0}+\mu\left(s_{1} \cup s_{2}\right) \Delta u \\
& -\left(\mu\left(s_{1}-s_{2}\right)+\frac{1}{2}\left(\left(\mu\left(s_{1} \cap s_{2}\right)+\left(1-\mu\left(s_{1} \cup s_{2}\right)\right)\right) x t\right.\right. \\
& -\left(\mu\left(s_{2}-s_{1}\right)+\frac{1}{2}\left(\left(\mu\left(s_{1} \cap s_{2}\right)+\left(1-\mu\left(s_{1} \cup s_{2}\right)\right)\right)(1-x) t,\right.\right.
\end{aligned}
$$


where $s_{1}-s_{2}$ means $s_{1} \cap s_{2}^{c}$. $u_{0}+\mu\left(s_{1} \cup s_{2}\right) \Delta u$ represents utility from reading gross of the transportation cost. The transportation cost depends on the composition of the articles covered by the aggregator, and is equal to the measure of articles from newspaper 1 multiplied by $x t$ plus the measure of articles from 2 multiplied by $(1-x) t$.

Using $\mu\left(s_{1} \cup s_{2}\right)=\mu\left(s_{1}\right)+\mu\left(s_{2}\right)-\mu\left(s_{1} \cap s_{2}\right)$ and $\mu\left(s_{i}-s_{j}\right)=\mu\left(s_{i}\right)-\mu\left(s_{1} \cap s_{2}\right)$, we can rewrite $U^{A g g}(x), U^{1}(x)$ and $U^{2}(x)$ as follows:

$$
\begin{aligned}
U^{A g g}(x) & =u_{0}-\frac{t}{2}+\mu\left(s_{1} \cup s_{2}\right) \Delta u+t\left(x-\frac{1}{2}\right)\left(\mu\left(s_{2}\right)-\mu\left(s_{1}\right)\right) ; \\
U^{1}(x) & =u_{0}-\frac{t}{2}+\mu\left(s_{1}\right) \Delta u+t\left(\frac{1}{2}-x\right) ; \\
U^{2}(x) & =u_{0}-\frac{t}{2}+\mu\left(s_{2}\right) \Delta u+t\left(x-\frac{1}{2}\right) .
\end{aligned}
$$

Hence, it is clear that a consumer located at $x=1 / 2$ loses nothing by choosing the aggregator; $U^{A g g}(1 / 2) \geq \max \left\{U^{1}(1 / 2), U^{2}(1 / 2)\right\}$. Consider now a consumer with location $x<1 / 2$. We have

$$
U^{A g g}(x)-U^{1}(x)=\underbrace{\left(\mu\left(s_{1} \cup s_{2}\right)-\mu\left(s_{1}\right)\right) \triangle u}_{\text {Benefit from higher quality }}-\underbrace{t\left(\frac{1}{2}-x\right)\left(1+\mu\left(s_{2}\right)-\mu\left(s_{1}\right)\right)}_{\text {Cost from higher preference mismatch }} .
$$

The benefit of using the aggregator instead of newspaper 1 is captured by the term $\left(\mu\left(s_{1} \cup s_{2}\right)-\mu\left(s_{1}\right)\right) \triangle u$, which represents surplus increase from consuming more high-quality content. This benefit comes with the cost of greater preference mismatch since, for a consumer with location $x<1 / 2$, the favorite newspaper is 1 . More precisely, the last term in (5) always has a negative sign for $x<1 / 2$ and represents the cost of using the aggregator.

More generally, the following lemma shows that newspapers are not directly in competition with each other.

Lemma 2. Newspapers are not directly in competition with each other: For any given $\left(s_{1}, s_{2}\right)$, there exists no $x \in[0,1]$ such that $\min \left\{U^{1}(x), U^{2}(x)\right\}>U^{\text {Agg }}(x)$.

Proof. To prove the lemma we consider two cases.

1) If $x<\frac{1}{2}$, then $U^{A g g}(x)>U^{2}(x)$ since $\mu\left(s_{2}\right)-\mu\left(s_{1}\right)<\frac{1}{2}$.

2) If $x>\frac{1}{2}$, then $U^{A g g}(x)>U^{1}(x)$ since $\mu\left(s_{1}\right)-\mu\left(s_{2}\right)<\frac{1}{2}$.

Let $x_{i}$ denote the location of the consumer who is indifferent between newspaper $i(i=1,2)$ and the aggregator. Then, for any $x<x_{1}$, we have $U^{1}(x)>U^{A g g}(x)$. This, together with Lemma 2 , implies $U^{1}(x)>U^{2}(x)$ for any $x<x_{1}$. Therefore, 1's market share is given by $x_{1}$. Similarly, 2's market share is given by $1-x_{2}$. Furthermore, $U^{A g g}(1 / 2) \geq \max \left\{U^{1}(1 / 2), U^{2}(1 / 2)\right\}$ means 
that $x_{1} \leq 1 / 2 \leq x_{2}$. Therefore, the aggregator's market share is $x_{2}-x_{1}$. The next lemma shows that each newspaper has a positive market share under A1.

Lemma 3. Under A1, for any given $\left(s_{1}, s_{2}\right)$ satisfying $\mu\left(s_{i}\right) \leq 1 / 2$ for $i=1,2$, the market shares of 1 and 2 are:

$$
\begin{aligned}
& 0<\alpha_{1}=\frac{1}{2}-\beta \frac{\mu\left(s_{2}\right)-\mu\left(s_{1} \cap s_{2}\right)}{1-\mu\left(s_{1}\right)+\mu\left(s_{2}\right)} \leq \frac{1}{2} \\
& 0<\alpha_{2}=\frac{1}{2}-\beta \frac{\mu\left(s_{1}\right)-\mu\left(s_{1} \cap s_{2}\right)}{1+\mu\left(s_{1}\right)-\mu\left(s_{2}\right)} \leq \frac{1}{2}
\end{aligned}
$$

The aggregator decreases the market share of the newspapers. Lemma 3 shows that for any $\left(s_{1}, s_{2}\right)$ satisfying $\mu\left(s_{i}\right) \leq 1 / 2$, the market share of a newspaper cannot be larger than $1 / 2$, whereas without the aggregator it is possible for a newspaper to have a market share larger than $1 / 2$ (although not in equilibrium). This result holds even when the quality of newspaper 1 , say, is maximal, i.e., $1 / 2$, and the quality of 2 is zero: in such a case the consumers located at $x \in(1 / 2,1]$ prefer the aggregator to newspaper 1 . By using the aggregator, they consume all the high-quality content of 1 , and, in the absence of high-quality content, they consume low-quality content from 2 half of the time.

The market share of each newspaper decreases in $\beta$, which means that the more loyal consumers are, the greater the newspapers' market shares. Holding $\left(\mu\left(s_{1}\right), \mu\left(s_{2}\right)\right)$ constant, increasing $s_{1} \cap s_{2}$ reduces the high-quality content available from the aggregator and increases the market share of both newspapers. In the extreme case of $s_{1}=s_{2}$, there is no room for the aggregator and each newspaper shares the whole market equally.

From Lemma 3, we can see the effect of quality, $\mu\left(s_{i}\right)$ with $i=1,2$, on the market share of, for instance, newspaper 1:

- $\alpha_{1}$ increases if newspaper $i(=1,2)$ increases its quality, $\mu\left(s_{i}\right)$, by investing in those issues which are also covered by $j(\neq i)$ too, i.e., by increasing $\mu\left(s_{1} \cap s_{2}\right)$.

- $\alpha_{1}$ decreases if newspaper $i(=1,2)$ increases its quality, $\mu\left(s_{i}\right)$, by investing in those issues which are not covered by $j(\neq i)$, i.e., by increasing $\mu\left(s_{i}-s_{j}\right)$.

In the subsequent analysis, it is important to understand the above effects of changes in quality $\mu\left(s_{i}\right)$ on 1's market share. If newspaper 1 increases its quality by investing in those issues covered by 2, this increases 1's market share, which seems to be standard. In contrast, if newspaper 1 increases its quality by investing in those issues not covered by 2, this reduces 1 's market share, which looks puzzling. To explain it, let us suppose that newspaper 1 increases its 
quality on an issue which 2 covers with low-quality and examine how this affects the utility of the consumer indifferent between newspaper 1 and the aggregator. Note first that the consumer can enjoy this quality increase regardless of whether she chooses newspaper 1 or the aggregator. But the quality increase reduces the consumer's transportation cost from choosing the aggregator while it does not affect the transportation cost from reading newspaper 1 . The reason is that on the issue, the probability for the aggregator to direct the consumer to the article of 1 is one after the quality increase while the probability was a half before the quality increase. This together with the fact the indifferent consumer is located at $x<1 / 2$ implies that newspaper 1 's market share decreases after the quality increase.

\subsection{Business-stealing vs. readership-expansion for given qualities}

Given $\left(s_{1}, s_{2}\right)$, newspaper $i$ 's profit is given by:

$$
\pi_{i}\left(s_{i}\right)=\alpha_{i}\left[1+\mu\left(s_{i}\right) \delta\right]+\delta\left(1-\alpha_{i}-\alpha_{j}\right)\left(\mu\left(s_{i}-s_{j}\right)+\frac{1}{2} \mu\left(s_{i} \cap s_{j}\right)\right)-c \mu\left(s_{i}\right)^{2} ;
$$

where $j \in\{1,2\}, j \neq i$. The term in the middle of the R.H.S. represents the revenue from the consumers directed by the aggregator.

The following proposition states that there exists no equilibrium in which the set of the common issues covered by 1 and $2, s_{1} \cap s_{2}$, is neither the maximum nor the minimum.

Proposition 2. Given $\mu\left(s_{i}\right)$ satisfying $0<\mu\left(s_{i}\right) \leq 1 / 2$ for newspaper $i \in\{1,2\}$, choosing $s_{i}$ such that $0<\mu\left(s_{1} \cap s_{2}\right)<\min \left(\mu\left(s_{1}\right), \mu\left(s_{2}\right)\right)$ is strictly dominated by choosing $s_{i}$ such that $\mu\left(s_{1} \cap s_{2}\right)=0$ or $\mu\left(s_{1} \cap s_{2}\right)=\min \left(\mu\left(s_{1}\right), \mu\left(s_{2}\right)\right)$. In other words, each newspaper is always better off choosing maximum or minimum differentiation.

The proof of Proposition 2 reveals that newspaper $i$ 's profit is convex with respect to $\mu\left(s_{1} \cap\right.$ $\left.s_{2}\right)$ : profit is therefore maximized at the corners. This result does not depend on A2 and holds for any arbitrary cost function. From Lemma 3 and the discussions following it we know that the aggregator's market share is minimized under minimum differentiation and maximized under maximum differentiation. Proposition 2 implies that newspaper $i$ finds it optimal either to "accommodate" the aggregator by maximum differentiation or to "fight" it with minimum differentiation.

Consider a given symmetric quality $\mu\left(s_{1}\right)=\mu\left(s_{2}\right)=\mu \in(0,1 / 2) \leq 1 / 2$. If newspaper $i$ uses the minimum differentiation strategy, the aggregator gets zero market share and hence each newspaper's profit is not affected by the presence of the aggregator. If $i$ uses instead the maximum differentiation strategy, each newspaper has the same market share $\left(\alpha_{1}=\alpha_{2}=\alpha=1 / 2-\beta \mu\right)$ and obtains identical profits $(\alpha[1+\mu \delta]+\delta(1-2 \alpha) \mu)$. The difference between a newspaper's 


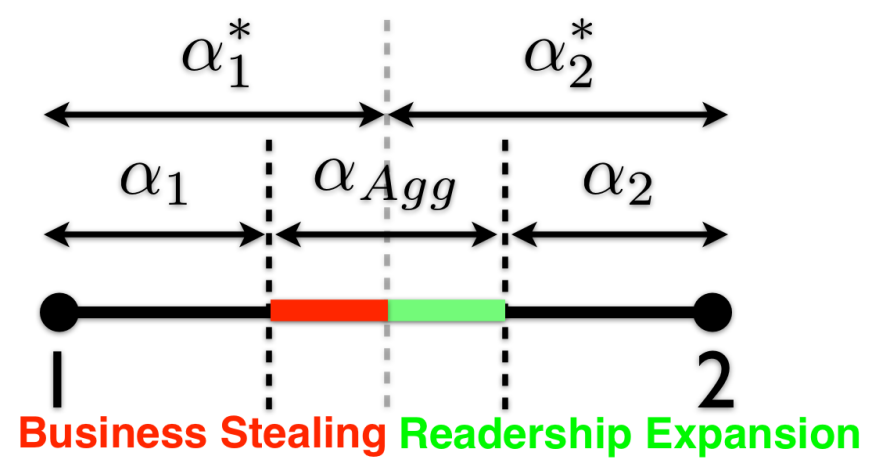

Figure 3: Business-stealing effect and readership-expansion effect from 1's point of view

profit under maximum differentiation and its profit in the absence of the aggregator (which is equal to the profit under minimum differentiation) is given by:

$$
\pi_{i}(\mu, \mu \mid \max )-\left.\pi_{i}(\mu, \mu)\right|_{\text {no aggregator }}=\underbrace{-\beta \mu * 1}_{\text {Business-stealing effect }}+\underbrace{\beta \mu * \delta \mu}_{\text {readership-expansion effect }}(=\beta \mu(\delta \mu-1)) .
$$

The first term on the R.H.S. of the above equation shows the business-stealing effect of the aggregator: the aggregator reduces the attention spent on the homepages of each newspaper by $\beta \mu * 1$. The second term on the R.H.S. shows the readership-expansion effect of the aggregator: the aggregator improves the match between attention and high-quality content, allowing each newspaper's high-quality content to reach more customers. Note that this readership expansion includes some customers who, without the aggregator, would read only the rival newspaper (See Figure 3). $\beta \mu * \delta \mu$ measures this increase in attention. From the previous discussion, we have:

Lemma 4. Consider any symmetric equilibrium candidate $\mu\left(s_{1}\right)=\mu\left(s_{2}\right)=\mu$ satisfying $0<\mu \leq$ $1 / 2$. The newspapers will use the maximum differentiation strategy (respectively, the minimum differentiation strategy) if $\delta \mu>1$ (respectively, if $\delta \mu<1$ ).

Although we have only considered the case of symmetric quality, the trade-off between the business-stealing effect and the readership-expansion effect is quite general. All other things being equal, as $\mu_{j}$ increases, the aggregator has a larger market share under maximal differentiation, and hence the readership-expansion effect is likely to dominate the business-stealing effect from $i$ 's point of view. As $\delta$ increases, the profit from high-quality content is more important relative to the profit from low-quality content, which likewise makes the readership-expansion effect more likely to dominate the business-stealing effect. More generally, Figure 4 describes, given 


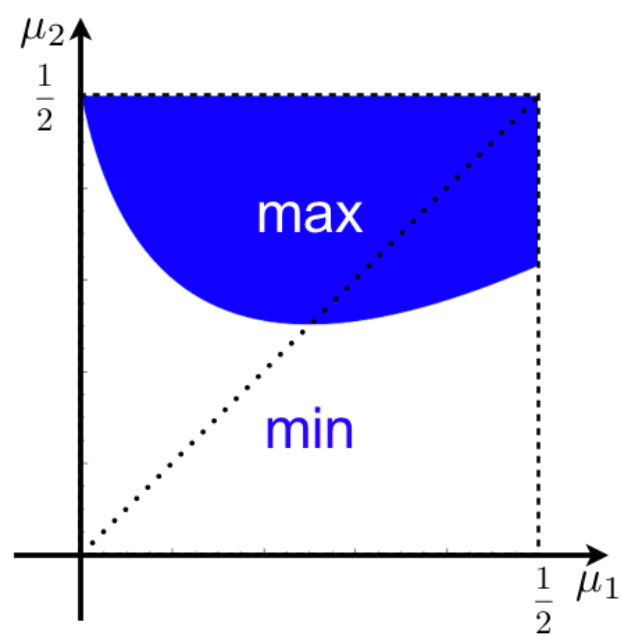

Figure 4: Best reply function of newspaper 1 given $\mu_{1}$, and $\mu_{2}$

$\left(\mu_{1}, \mu_{2}\right) \in(0,1 / 2]^{2}$, under what conditions minimum or maximum differentiation is the optimal strategy for newspaper 1 .

Remark: The previous discussion shows that the presence of the aggregator can never decrease a newspaper's profit, given symmetric quality: each newspaper can always reduce the aggregator's market share to zero by using the minimum differentiation strategy and thereby obtain the profit it received without the aggregator. This is a consequence of the fact that we consider only two newspapers. On the contrary, if there are many newspapers and some of them use maximum differentiation, a single newspaper cannot reduce the market share of the aggregator to zero. After completely characterizing the outcomes for two newspapers, we extend the model to allow the aggregator to provide content from third-party news sites different from the two newspapers (see Section 6.3).

As a consequence of Proposition 2, there are two equilibrium candidates, one with minimum differentiation and the other with maximum differentiation. The following subsections address each one in turn.

\subsection{Minimum differentiation (no specialization) equilibrium}

In this subsection, we study the existence of the equilibrium in which the newspapers choose minimum differentiation, or equivalently $s_{1}=s_{2}$. Let $\left(\mu_{1}^{m}, \mu_{2}^{m}\right)$ denote the equilibrium qualities under the minimum differentiation strategy. We have:

Proposition 3. Under A1-A3, there are $\left(\underline{\delta}^{m}, \bar{\delta}^{m}\right)$ satisfying $0<\underline{\delta}^{m} \leq \bar{\delta}^{m}$ such that for any $\delta>\bar{\delta}^{m}$ there exists no symmetric equilibrium in which newspapers invest in the same set of 


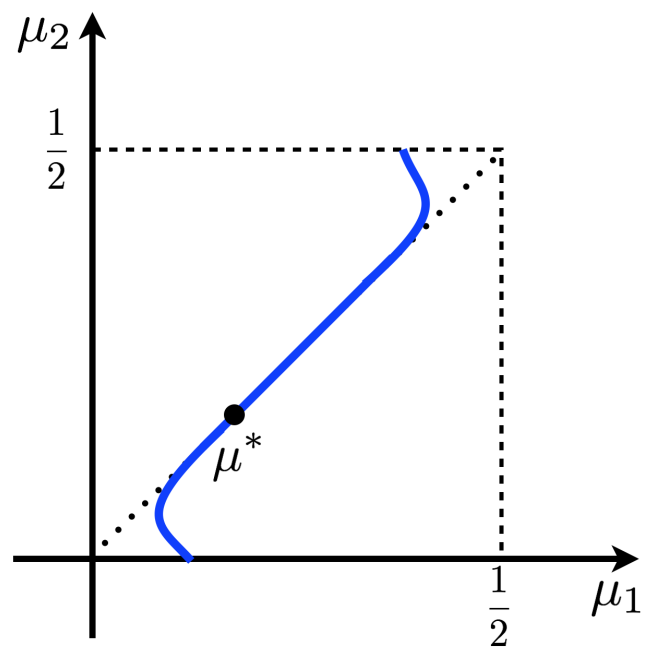

Figure 5: Best reply function of newspaper 1 given min differentiation

issues: for any $\delta \leq \underline{\delta}^{m}$ there exist multiple symmetric equilibria in which newspapers invest in the same set of issues:

$\begin{array}{llrl}\text { (i) } \mu_{1}^{m}=\mu_{2}^{m}=\mu^{m} \in\left[\frac{\delta}{4 c-\delta \beta}, \frac{1}{2}\right] & \text { if } & \frac{\delta}{2}+\frac{\delta \beta}{4}+\frac{\beta}{2}<c \leq \frac{\delta}{2}+\frac{\delta \beta}{4}+\beta ; \\ \text { (ii) } \mu_{1}^{m}=\mu_{2}^{m}=\mu^{m} \in\left[\frac{\delta}{4 c-\delta \beta}, \frac{\delta+2 \beta}{4 c-\delta \beta}\right] & \text { if } & c>\frac{\delta}{2}+\frac{\delta \beta}{4}+\beta .\end{array}$

Corollary 1. $\mu^{m}=\mu^{*}$ (where $\mu^{*}$ is the quality which arises in the equilibrium without the aggregator) is one of the minimum differentiation equilibria.

The intuition behind this result is simple. If the revenue from high-quality content is high enough, each newspaper has an incentive to use the maximum differentiation strategy: the readership-expansion effect dominates the business-stealing effect. On the contrary, when the revenue from high quality content is low enough, the business-stealing effect dominates the readership-expansion effect and each newspaper uses the minimum differentiation strategy. Since any equilibrium quality $\mu_{1}^{m}$ is a best response to $\mu_{2}^{m}$ for the interval of equilibrium qualities described in Proposition 3, the best reply curve has a slope of 45 degree (see also Figure 5 ). Hence, quality choices are strategic complements over this interval. The reason is that given $\mu\left(s_{2}\right)=\mu_{2}^{m}$, newspaper 1 finds it optimal to "fight" the aggregator by choosing $s_{1}=s_{2}$, which leaves zero market share to the aggregator. More precisely, conditional on using the minimum differentiation strategy, newspaper 1's profit increases when $\mu_{1}$ increases up to $\mu_{2}^{m}$ and decreases when when $\mu_{1}$ increases beyond $\mu_{2}^{m}$. Figure 5 also shows that the equilibrium quality without the aggregator, $\mu^{*}$, belongs to the interval of equilibrium qualities under the minimum differentiation strategy. 


\subsection{Maximum differentiation (specialization) equilibrium}

In this subsection, we study the equilibrium candidate with maximum differentiation. The profit of newspaper $i \in\{1,2\}$ conditional on maximum differentiation is given by:

$$
\pi_{i}\left(s_{i} \mid \max \right)=\frac{1}{2}+\frac{\delta}{2} \mu_{i}-\beta \frac{\mu_{j}}{1+\mu_{j}-\mu_{i}}+\delta \beta \frac{\mu_{i}^{2}}{1+\mu_{i}-\mu_{j}}-c \mu_{i}^{2} .
$$

Let $\left(\mu_{1}^{M}, \mu_{2}^{M}\right)$ denote the equilibrium qualities under the maximum differentiation strategy. Figure 6(a) shows the best reply conditional on both newspapers' using the maximum differentiation strategy. It shows that the curve crosses the 45 degree line only once and has a positive slope when (and after) crossing it. More precisely, we have

$$
\frac{\partial \pi_{i}}{\partial \mu_{i} \partial \mu_{j}}=-\beta \frac{1-\mu_{i}-\mu_{j}}{\left(1-\mu_{i}+\mu_{j}\right)^{2}}+2 \delta \beta \frac{\mu_{i}\left(1-\mu_{j}\right)}{\left(1+\mu_{i}-\mu_{j}\right)^{3}}
$$

which is positive for $\delta \mu_{i} \geq 1 / 2$. Since $\delta \mu^{M}>1$ holds from Lemma 4, quality choices are strategic complements for quality above $\mu^{M}$ and for quality just below (and close to) $\mu^{M}$.

Lemma 5. In the presence of the aggregator, suppose that a symmetric equilibrium with maximum differentiation strategy $\mu_{1}^{M}=\mu_{2}^{M}=\mu^{M}$ exists. Then, there exists some $\mu^{\prime}$ satisfying $\mu^{\prime}<\mu^{M}$, such that conditional on newspaper $i$ using the maximum differentiation strategy, an increase in $\mu_{j}$ induces an increase in $\mu_{i}$ for any $\mu_{j} \geq \mu^{\prime}$. Therefore, newspapers' quality choices $\left(\mu_{1}, \mu_{2}\right)$ are strategic complements for $\mu_{j} \geq \mu^{\prime}$.

When newspaper 1 uses the maximum differentiation strategy, an increase in $\mu_{2}$ expands the market share of the aggregator and hence increases the readership-expansion effect. This increased readership-expansion effect in turn increases the marginal revenue from an increase in $\mu_{1}$, which makes quality choices strategic complements. Figure 6(b) shows that this property holds even when a newspaper is not restricted to the maximum differentiation strategy, since it is optimal for $i$ to use this strategy for $\mu_{j}$ larger than some threshold (see Figure 4 ).

Proposition 4. Under A1-A3, there exists a threshold $\bar{\delta}^{M}>0$ such that for any $\delta \geq \bar{\delta}^{M}$ there is a unique symmetric equilibrium, $\mu_{1}^{M}=\mu_{2}^{M}=\mu^{M}$, in which newspapers invest in disjoint sets of issues. This $\mu^{M}$ is

(i) $\frac{1}{2}$

(ii) $\frac{(-\beta+2 \delta \beta-2 c)+\sqrt{(-\beta+2 \delta \beta-2 c)^{2}+2 \delta^{2} \beta}}{2 \delta \beta}$ if $c>\frac{\delta}{2}-\frac{\beta}{2}+\frac{3}{4} \delta \beta$

Moreover, there exists another threshold $\underline{\delta}^{M} \in\left(0, \bar{\delta}^{M}\right)$ such that, for any $\delta<\underline{\delta}^{M}$, there exists no equilibrium with maximum differentiation. 


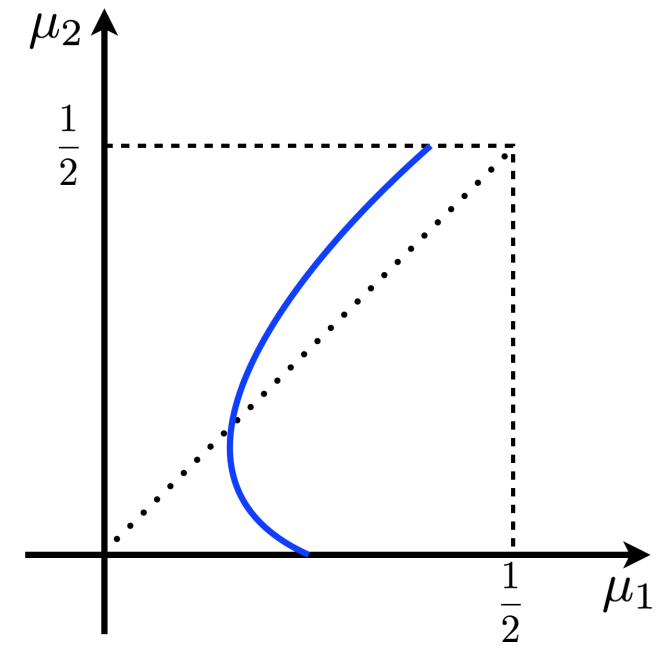

(a) Given max differentiation

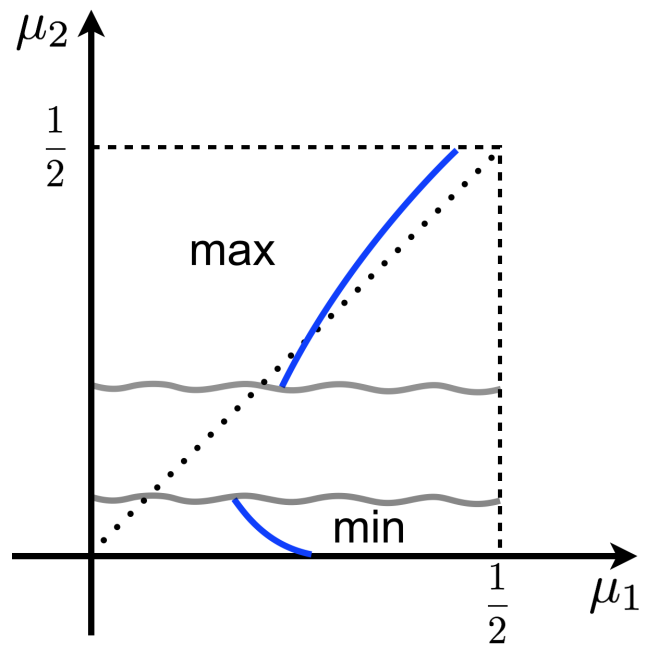

(b) Actual best reply function given $\delta>\bar{\delta}^{M}$

Figure 6: Best reply functions of newspaper 1

It follows from Lemma 4 that $\delta>2$ is a necessary condition for the existence of the maximum differentiation equilibrium (equivalently called, the specialization equilibrium). One can check that $\mu^{M}$ is increasing in $\delta$ : as the revenue from high-quality content increases, newspapers have more incentive to invest in quality. Moreover, if consumers are less loyal (i.e., as $\beta$ increases), competition becomes tougher and hence the newspapers invest more in quality.

\subsection{Equilibrium refinement with opt-out option}

In this subsection we analyze the following two-stage game to refine the equilibria obtained in the previous analysis:

- Stage 1: each newspaper $i$ simultaneously decides whether to opt out or not and chooses $s_{i}$.

- Stage 2: each consumer chooses one among the two newspapers and the aggregator.

Note that if newspaper $i$ opts out, it breaks the link with the aggregator and hence the aggregator has content only from $j$ : in this case we break the tie by assuming that consumers prefer using newspaper $j$ to the aggregator. ${ }^{21}$ Then, we always have an equilibrium in which

\footnotetext{
${ }^{21}$ This tie-breaking makes sense since the navigation between the aggregator's site and newspaper $j$ 's site is less seamless than the navigation within newspaper $j$ 's site.
} 
all newspapers opt out. In this opting out equilibrium, each newspaper chooses the quality $\mu^{*}$. This equilibrium trivially exists regardless of the number of competing newspapers.

We now check how the opt-out option affects the equilibria under minimum differentiation. Given $\mu\left(s_{j}\right)=\mu^{m}$, does the possibility to opt out induce newspaper $i$ to deviate from choosing $s_{i}=s_{j}$ ? The answer is yes for any $\mu^{m}$ different from $\mu^{*}$. Note first that in the minimum differentiation equilibrium candidate, each newspaper gets the profit it would obtain without the aggregator from symmetric quality $\mu^{m}$. Therefore, as long as $\mu^{m}$ is different from $B R_{i}^{N}\left(\mu^{m}\right)$, i.e., newspaper $i$ 's best response to $\mu\left(s_{j}\right)=\mu^{m}$ without the aggregator, newspaper $i$ has an incentive to opt out and to choose $B R_{i}^{N}\left(\mu^{m}\right)$. Since we have a unique equilibrium without the aggregator, $\mu^{m}=B R_{i}^{N}\left(\mu^{m}\right)$ holds if and only if $\mu^{m}=\mu^{*}$. This implies that only $\mu^{m}=\mu^{*}$ survives the introduction of an opt-out option.

In the maximum differentiation equilibrium, this does not necessarily hold. For a given $\mu\left(s_{1}\right)=\mu\left(s_{2}\right)=\mu^{M}$, from (9), each firm gets a higher profit in the specialization equilibrium than without the aggregator. If $i$ opts out for given $\mu\left(s_{j}\right)=\mu^{M}$ satisfying $\delta \mu^{M}>1$, its best response is $B R_{i}^{N}\left(\mu^{M}\right)$. It is possible that the profit from this deviation is lower than the equilibrium profit. To see this, note that without the aggregator, an increase in $\mu_{j}$ reduces the marginal profit of $i$ and that $\mu^{M}>\mu^{*}$ (see Proposition 6).

Therefore, the introduction of an opt-out option leads to a sharp prediction: the presence of the aggregator either leads to no change or to the specialization equilibrium. In summary:

Proposition 5. When newspapers can opt out,

(i) There always exists an equilibrium in which every newspaper opts out and chooses the equilibrium quality without the aggregator $\left(\mu_{1}=\mu_{2}=\mu^{*}\right)$.

(ii) Among all equilibria with minimum differentiation, only the equilibrium quality without the aggregator survives the introduction of an opt-out option.

(iii) The maximum differentiation equilibrium survives the introduction of an opt-out option if the deviation to "opting out and choosing $\mu_{i}=B R_{i}^{N}\left(\mu^{M}\right)$ " is not profitable.

\section{Comparison: quality, consumer surplus, profit and welfare}

In this section, we study how the aggregator affects quality, consumer surplus, profits and welfare. From Proposition 5, we compare the equilibrium without the aggregator with the specialization equilibrium. We first address quality:

Proposition 6. Under A1-A3, the quality of newspapers is higher in the maximum differentiation equilibrium than in the equilibrium without the aggregator, i.e., $\mu^{M}>\mu^{*}$. 
Note that the existence of the maximum differentiation equilibrium requires $\delta$ large enough (i.e., $\delta \mu^{M}>1$ ). In the presence of the aggregator, for $\delta$ large enough, $\mu_{1}=\mu_{2}=\mu^{*}$ is not an equilibrium. This is because the readership-expansion effect dominates the business-stealing effect and hence each newspaper finds it optimal to respond by increasing quality above $\mu^{*}$ and using maximum differentiation. Furthermore, quality choices are strategic complements. Therefore, newspapers choose $\mu_{1}=\mu_{2}=\mu^{M}>\mu^{*}$.

We now study how the aggregator affects consumer surplus and newspapers' profits. Consumer surplus and the profit of each newspaper in the absence of the aggregator are given by:

$$
\begin{gathered}
C S^{*}=\int_{0}^{\frac{1}{2}}\left(\mu^{*} \triangle u+u_{0}-x t\right) d x+\int_{\frac{1}{2}}^{1}\left(\mu^{*} \triangle u+u_{0}-(1-x) t\right) d x=\mu^{*} \triangle u+u_{0}-\frac{t}{4} ; \\
\pi^{*}=-c \mu^{* 2}+\frac{\delta}{2} \mu^{*}+\frac{1}{2} .
\end{gathered}
$$

Since the aggregator induces each newspaper to choose a higher quality, this increases every consumer's surplus. Even if a consumer continues to use her preferred newspaper, she benefits from the quality increase. In addition, she has the option of using the aggregator.

The profit of each newspaper in the specialization equilibrium is $\pi^{M}=\alpha^{M}\left[1+\delta \mu^{M}\right]+(1-$ $\left.2 \alpha^{M}\right) \delta \mu^{M}-c \mu^{M^{2}}$, where $\alpha^{M}$ is the share of each newspaper and, from (6) and (7), is equal to $\frac{1}{2}-\beta \mu^{M}$. Thus, each newspaper's profit is

$$
\pi^{M}=\mu^{M^{2}}(\delta \beta-c)+\mu^{M}\left(-\beta+\frac{\delta}{2}\right)+\frac{1}{2} .
$$

The presence of the aggregator increases each newspaper's profit if and only if

$$
\mu^{M^{2}}(\delta \beta-c)+\mu^{M}\left(-\beta+\frac{\delta}{2}\right)+\frac{1}{2} \geq \frac{1}{2}+\frac{\delta}{2} \mu^{*}-c \mu^{*^{2}} .
$$

Proposition 7. Suppose that the presence of the aggregator leads to the maximum differentiation equilibrium. Then, in the presence of the aggregator:

(i) Every consumer gets a higher surplus.

(ii) The profits of newspapers increase if the cost is low enough, and decrease otherwise. More precisely, there exists $\hat{c}$ such that $\pi^{M}<\pi^{*}$ for all $c>\hat{c}$ and $\pi^{M}>\pi^{*}$ for all $c<\hat{c}$.

(iii) Social welfare is higher.

The profits of the newspapers can be lower in the specialization equilibrium than in the equilibrium without the aggregator. More generally, Proposition 7 shows that whether the profits increase or decrease depends on the cost level $c$. As we noted in Section 4.2, for a given quality, the 
aggregator cannot decrease each newspaper's profit. Furthermore, from equation (9), the profit in the maximum differentiation equilibrium (gross of the investment cost) strictly increases with $\mu^{M}$. This implies that the aggregator increases each newspaper's profit if the investment cost does not increase too much (i.e., if $c$ is low enough).

Note that the relevant cost to consider is actually $c / \varpi$ where $\varpi$ is advertising revenue per unit of attention, previously normalized to one. If the Internet creates advertising congestion (Anderson, Foros, Kind, and Peitz, 2012) by expanding advertising possibilities and thereby reduces $\varpi$, this increases $c / \varpi$. This suggests that the aggregator is likely to reduce the profits of newspapers, which may explain why the current debate on news aggregators is so heated.

Finally, we show that the presence of the aggregator increases social welfare. We proceed in two steps. First, for given symmetric quality (for instance, $\mu^{*}$ ), the presence of the aggregator increases social welfare. This is because both consumer surplus increases, and total traffic to the newspapers and the aggregator increases. The total traffic increases since traffic to the homepages is constant, while traffic to high-quality articles increases thanks to the aggregator. Second, we can show that, in the presence of the aggregator, the newspapers choose too low-quality from a social point of view, which implies that the increase in quality from $\mu^{*}$ to $\mu^{M}$ (while maintaining maximum differentiation) is welfare-improving. To see this, consider a marginal change in $\mu_{1}$ for any given $\mu_{2}$. We have

$$
\frac{\partial S W}{\partial \mu_{1}}=\frac{\partial \pi_{1}}{\partial \mu_{1}}+\frac{\partial \pi_{2}}{\partial \mu_{1}}+\frac{\partial \pi_{A}}{\partial \mu_{1}}+\frac{\partial C S}{\partial \mu_{1}}
$$

where $\pi_{A}$ is the profit of the aggregator. $\frac{\partial C S}{\partial \mu_{1}}>0$ is obvious and we can show

$$
\frac{\partial \pi_{2}}{\partial \mu_{1}}+\frac{\partial \pi_{A}}{\partial \mu_{1}}>0
$$

From Lemma 3, as $\mu_{1}$ increases, newspaper 1's market share decreases under maximum differentiation. This implies that as $\mu_{1}$ increases, total traffic to the homepages of newspaper 2 and the aggregator increases. Furthermore, it also implies that the traffic to the high-quality articles of 2 increases. Therefore, an increase in $\mu_{1}$ generates positive externalities on the joint profit of newspaper 2 and the aggregator, and on consumers. Hence, if $\frac{\partial \pi_{1}}{\partial \mu_{1}}=0$, then $\frac{\partial S W}{\partial \mu_{1}}>0$.

\section{Extensions}

In this section, we provide results for four extensions: asymmetric issues, imperfect certification technology, third-party content and paywall. These extensions make the model closer to the real world and show the robustness of our main results. 


\subsection{Asymmetric issues}

In the baseline model, we assumed that all issues are of equal importance in terms of probability of click, which is not realistic. We discuss here what happens if we assume that some issues (such as those covering major events) have a higher probability of click than the other issues. Let $S \equiv S_{A} \cup S_{B}$ where $S_{A} \cap S_{B}=\varnothing$. Given that an article has high-quality, the probability for a reader to click its link is $p_{A}\left(p_{B}\right)$ if the issue belongs to $S_{A}\left(S_{B}\right)$, with $p_{A}>p_{B}$. The probability of click is zero for low-quality articles. If the difference between $p_{A}$ and $p_{B}$ is large enough and the measure of $S_{A}$ is not too large, regardless of the presence of the aggregator, both newspapers will cover all issues in $S_{A}$ with high-quality (i.e., both newspapers cover major events with high-quality articles). Therefore, we can interpret $u_{0}$ as the utility from reading a home page and high-quality articles covering major events, which makes the assumption $u_{0}>t$ more easily satisfied. In addition, assumption A2 is relaxed as follows:

$$
C\left(\mu\left(s_{i}\right)\right)= \begin{cases}\infty & \mu\left(s_{i}\right)>\mu\left(S_{A}\right)+\mu\left(S_{B}\right) / 2 \\ c \mu\left(s_{i}\right)^{2} & \mu\left(s_{i}\right) \leq \mu\left(S_{A}\right)+\mu\left(S_{B}\right) / 2 .\end{cases}
$$

Since this extension is isomorphic to the baseline model, we can conclude that the aggregator induces newspapers to specialize in the coverage of the issues belonging to $S_{B}$ (i.e., those which are not major events of the day but have important social concerns such as climate change, income inequality etc.) and to increase the quality of the articles on these issues.

\subsection{Imperfect certification technology}

When each newspaper provides an article of different quality on a given issue, let $(1+\Delta P) / 2$ (respectively, $(1-\Delta P) / 2$ ) represent the probability for the aggregator to provide the link to the high-quality article (respectively, to the low-quality article) where $\Delta P \in[0,1] . \Delta P=1$ corresponds to the case of perfect certification technology in the baseline model. We below briefly present the most interesting results; the detailed analysis can be found in the supplementary materials.

In this extension of the baseline model, there exists a unique threshold $\hat{P}$ in $(0,1)$ such that $\partial \alpha_{1} / \partial \mu_{1} \leq 0$ if and only if $\Delta P \geq \hat{P} \cdot{ }^{22}$ What is even more interesting is that the aggregator's market share always increases with $\mu_{i}$ (for $\left.i=1,2\right): \partial\left(1-\alpha_{1}-\alpha_{2}\right) / \partial \mu_{i} \geq 0$ (the inequality is strict for $\Delta P>0$ ). For instance, for $\Delta P>0$ small, even if the aggregator loses consumers to newspaper 1 after the latter's quality increase, the aggregator steals more consumers from

\footnotetext{
${ }^{22}$ For instance, suppose $\Delta P=0$ and that newspaper 1 replaces a low-quality article with a high-quality one. Then, a consumer can enjoy this quality increase with probability equal to one by choosing newspaper 1 but only with probability equal to $1 / 2$ by choosing the aggregator. So 1's market share increases with its quality.
} 
newspaper 2. This is because a consumer cannot enjoy the quality increase by reading 2 but can enjoy it by choosing the aggregator.

Proposition 2 extends to the imperfect certification technology since the profit of a newspaper is convex in $\mu_{12}$. Lemma 4 also extends such that in a symmetric equilibrium with $\mu_{1}=\mu_{2}=\mu$, both newspapers use the maximum differentiation strategy (respectively, the minimum differentiation strategy) if $\Delta P \delta \mu>1$ (respectively, if $\Delta P \delta \mu<1$ ). Proposition 5 on equilibrium selection based on opting out still applies to $\Delta P \in[0,1]$. When we compare the maximum differentiation equilibrium with the equilibrium without the aggregator by using the necessary condition $\Delta P \delta \mu^{M}>1$, we find again that the presence of the aggregator increases the quality (Proposition 6). Moreover, the equilibrium quality increases with $\Delta P$ as noisier certification weakens the readership-expansion effect.

Finally, we find that the effect of $\Delta P$ on newspapers' profits in the maximum differentiation equilibrium is ambiguous. However, as the aggregator's certification technology becomes more accurate, the readership-expansion effect is more likely to dominate the business-stealing effect and this tends to increase newspapers' profits. This finding offers a possible explanation for newspapers' complaint against Google News: they may find Google's algorithm to select news articles too noisy, resulting in low profits for them.

\subsection{Content from third-party providers}

We believe what is happening in the online world can be represented by the specialization equilibrium. However, one may argue that the model does not reflect the real world since each newspaper has so much market power that it can unilaterally eliminate the aggregator by opting out. In the real world, a single newspaper has very little effect on the aggregator since the aggregator contains content from many news outlets. ${ }^{23}$ In particular, there are many small news sites which would receive very negligible traffic in the absence of the aggregator. Therefore, these sites have strong incentives to use "the maximum differentiation and opt-in strategy" in order to attract traffic from the aggregator. In order to capture this heterogeneity among news sites in our model, and to show the robustness of our main results, we introduce one important modification: by using the aggregator, consumers can get utility $u_{T}$ generated from the aggregation of content from numerous small third party providers $;{ }^{24}$ but we maintain the assumption of perfect certification. Therefore, even if the two newspapers opt out, a consumer can get a utility equal to $u_{T}$ from using the aggregator. However, in the absence of the aggregator, it is impossible for a consumer to obtain $u_{T}$ from the numerous small third-party providers. This implies that the introduction of $u_{T}$ does not affect the analysis of the case without aggregator.

\footnotetext{
${ }^{23}$ Google News indexes content from 25000 news outlets.

${ }^{24}$ Although $u_{T}$ can depend on a consumer's ideological taste, we abstract from this dimension for simplicity.
} 
In the presence of the aggregator, the utility that a consumer located at $x$ obtains from the aggregator is given by:

$$
U^{A g g}(x)=\mu\left(s_{1} \cup s_{2}\right) \Delta u+u_{0}-t x \mu\left(s_{1}\right)-t(1-x) \mu\left(s_{2}\right)-\frac{t}{2}\left(1-\mu\left(s_{1}\right)-\mu\left(s_{2}\right)\right)+u_{T} .
$$

The market shares of the newspapers are given as follows:

$$
\begin{aligned}
& \alpha_{1}=\frac{1}{2}-\frac{1}{t} \frac{\left(\mu\left(s_{2}\right)-\mu\left(s_{1} \cap s_{2}\right)\right) \Delta u+u_{T}}{1-\mu\left(s_{1}\right)+\mu\left(s_{2}\right)} ; \\
& \alpha_{2}=\frac{1}{2}-\frac{1}{t} \frac{\left(\mu\left(s_{1}\right)-\mu\left(s_{1} \cap s_{2}\right)\right) \Delta u+u_{T}}{1-\mu\left(s_{2}\right)+\mu\left(s_{1}\right)} .
\end{aligned}
$$

We focus on the case in which the utility form third-party content is sufficiently important:

A4: $u_{T} \geq \frac{\Delta u}{2} \max \left\{1, \frac{3}{\delta}\right\}$.

To avoid corner solutions under A4 (i.e., to guarantee a positive market share for each newspaper), we should modify A1 as follows.

A1': $4 u_{T}<t$

A1' puts an upper bound on $u_{T}$. Hence, under A1' and A4, depending on the parameter values, the equilibrium market share of the aggregator can vary from (close to) zero to (close to) one.

Proposition 8. Suppose that the utility from third-party content is high enough (i.e., A4 holds). Under A1', A2, A3;

(i) For any $\left(\mu\left(s_{1}\right), \mu\left(s_{2}\right)\right) \in[0,1 / 2]^{2}$, maximum differentiation, $\mu\left(s_{1} \cap s_{2}\right)=0$, is a dominant strategy for each newspaper.

(ii) For any $\delta>0$, newspapers' quality choices $\left(\mu\left(s_{1}\right), \mu\left(s_{2}\right)\right)$ are strategic complements.

(iii) For any $\delta>0$, there is a unique symmetric equilibrium, $\mu\left(s_{1}\right)=\mu\left(s_{2}\right)=\mu^{T}$, where newspapers invest on disjoint set of issues, $\mu\left(s_{1} \cap s_{2}\right)=0$. There are two thresholds of $\delta$ such that $\mu^{T}=0$ for all $\delta \leq \underline{\delta}^{T}$ and $\mu^{T}=\frac{1}{2}$ for all $\delta \geq \bar{\delta}^{T}\left(>\underline{\delta}^{T}\right)$. For $\delta \in\left[\underline{\delta}^{T}, \bar{\delta}^{T}\right]$, $\mu^{T}$ strictly increases with $\delta$.

When the utility from third-party content is high enough, the aggregator already has a nonnegligible market share, independent of what a single newspaper does. Therefore, this induces each newspaper to accommodate the aggregator by adopting the maximum differentiation strategy, such that the minimum differentiation equilibrium does not exist whereas the maximum differentiation equilibrium exists for all $\delta>0$. As we have seen before, this implies that newspapers' quality choices $\left(\mu\left(s_{1}\right), \mu\left(s_{2}\right)\right)$ are strategic complements for all $\delta>0$. Furthermore, the 
equilibrium quality is increasing in $\delta$ such that the presence of the aggregator can increase or decrease quality with respect to the case without the aggregator. If $\delta$ is small (respectively, large), the business-stealing effect is large (respectively, small) relative to the readership-expansion effect.

Therefore, the value of $\delta$ is the key parameter determining whether the aggregator increases or reduces the quality of newspapers. Even if there have not been any empirical studies directly estimating $\delta$, we think that the studies by Athey and Mobius (2012) and Chiou and Tucker (2012) allow us to pin down a lower bound of $\delta$. Athey and Mobius (2012) study a natural experiment in which Google News introduces news from local outlets for readers who enter their zip code. They find that after adding content from new local outlets to Google News, traffic increases not only to these new outlets but also to the old (local and non-local) outlets that have been indexed by Google News. Chiou and Tucker (2012) exploit a contract dispute which led Google News to remove the content from Associated Press (AP). They show that the presence of the AP content on Google News would have increased traffic to the news sites indexed by Google News, which are not necessarily members of the AP network. Therefore, we can infer from these papers that an increase in the third-party content $u_{T}$ would increase traffic to the two newspapers, for a given equilibrium quality of the newspapers, implying

$$
\left.\frac{\partial \pi^{T}}{\partial u_{T}}\right|_{\mu^{T}=c s t}>0 \Leftrightarrow \delta \mu^{T}>1
$$

where $\pi^{T}$ is the profit of each newspaper and is given by:

$$
\pi^{T}=\frac{1}{2}+\frac{\delta}{2} \mu^{T}+\frac{1}{t}\left(\mu^{T} \Delta u+u_{T}\right)\left(\delta \mu^{T}-1\right)-c \mu^{T^{2}}
$$

This means that the readership-expansion effect is larger than the business-stealing effect at equilibrium:

$$
\begin{aligned}
\pi_{i}^{T}(\mu, \mu \mid \max )-\left.\pi_{i}(\mu, \mu)\right|_{\text {no aggregator }} & =\underbrace{-\left(\beta \mu+\frac{u_{T}}{t}\right) * 1}_{\text {Business-stealing effect }}+\underbrace{\left(\beta \mu+\frac{u_{T}}{t}\right) * \delta \mu}_{\text {Readership-expansion effect }} \\
& =\left(\beta \mu+\frac{u_{T}}{t}\right)(\delta \mu-1)>0,
\end{aligned}
$$

where $\beta \mu+\frac{u_{T}}{t}$ is the market share lost by each newspaper to the aggregator. For $\delta \mu^{T}>1$, we can show that the aggregator increases quality and that each newspaper has no incentive to opt out in equilibrium.

Proposition 9. Consider the model with the third-party content and assume A1', A2-A4. If $\delta \mu^{T}>1$ : 
(i) The presence of the aggregator increases the quality of newspapers, $\mu^{T} \geq \mu^{*}$;

(ii) When the aggregator is present, each newspaper has no incentive to opt out;

(iii) The presence of the aggregator increases consumer surplus and social welfare.

The result that each newspaper has no incentive to opt out is proved in the Appendix. To obtain the result on consumer surplus and social welfare, we can apply the same logic used in Section 5 .

\subsection{Paywall}

So far we assumed that advertising is the only source of revenue for newspapers. In this subsection, we take the model of Section 6.3 and allow each newspaper to charge a price. The reason we choose the model of Section 6.3 is to provide a more realistic scenario in which consumers can still enjoy some content through the aggregator even if the two newspapers erect paywalls. We assume that prices cannot be strictly negative.

In the presence of the aggregator, we find a sufficient condition for each newspaper to find zero price profit-maximizing. For this purpose, we analyze the following three-stage game:

- Stage 1: each newspaper $i$ simultaneously chooses $s_{i}$.

- Stage 2: each newspaper $i$ simultaneously chooses the price, $p_{i}$.

- Stage 3: each consumer chooses one among the two newspapers and the aggregator.

We assume that upon choosing a positive price, a newspaper blocks any incoming traffic from the aggregator. We have:

Proposition 10. Consider the model with the third-party content. In the presence of the aggregator, for any given pair of quality, $\left(\mu\left(s_{1}\right), \mu\left(s_{2}\right)\right) \in R_{+}^{2}$, it is a dominant strategy for each newspaper $i(i=1,2)$ to choose $p_{i}=0$ if $t \leq \frac{4}{3}$.

The proposition shows a very intuitive result: if competition among newspapers is strong enough, each newspaper finds zero price profit-maximizing. It also explains why newspapers with market power such as Financial Times or Wall Street Journal want to erect paywall.

In the case of the three-stage game without the aggregator, we study the symmetric equilibrium in which both newspapers choose the same quality $\mu^{P}$ and charge a strictly positive price $p^{P}$. We have:

Proposition 11. Suppose that there is no aggregator.

(i) There exists a symmetric equilibrium in which both newspapers choose the same quality $\mu^{P}=\frac{\Delta u+\delta}{6 c}$ and charge a strictly positive price $p^{P}$ if $p^{P}=t-1-\delta \mu^{P}>0$. 
(ii) If $t \leq \frac{4}{3}$, the newspapers choose a higher quality without paywall than with paywall (i.e., $\left.\mu^{*}>\mu^{P}\right)$.

(iii) If $t \leq \frac{4}{3}$ and $2 \Delta u>\delta$, then the newspapers' profits are higher with paywall than without paywall.

The two propositions show that the aggregator may make the existence of paywall equilibrium more difficult in the sense that the equilibrium with paywalls can exist without the aggregator but does not exist with the aggregator. This happens if $t \leq \frac{4}{3}$. In addition, the last proposition shows that without the aggregator, paywalls soften quality competition such that newspapers choose lower quality and earn higher profits than without paywalls under the reasonable assumption $2 \Delta u>\delta$ (explained below). Therefore, our result that the aggregator increases newspapers' quality is robust to allowing for paywalls as long as competition between the newspapers is fierce enough. Our finding also provides another explanation for why newspapers complain about Google News: news aggregator intensifies competition among newspapers such that it is more difficult to erect paywalls.

We below provide an intuition for why paywalls soften quality competition. We have:

$$
\begin{aligned}
\pi_{1} & =\alpha_{1}\left(1+\delta \mu_{1}+p_{1}\right)-c \mu_{1}^{2} \\
\alpha_{1} & =\frac{1}{2}+\frac{\left(\mu_{1}-\mu_{2}\right) \Delta u+\left(p_{2}-p_{1}\right)}{2 t} \\
p_{i} & =t+\frac{\Delta u}{3}\left(\mu_{i}-\mu_{j}\right)-1-\frac{2 \delta \mu_{i}}{3}-\frac{\delta \mu_{j}}{3} .
\end{aligned}
$$

When we isolate how a change in $\mu_{1}$ affects 1 's profit through changes in $\left(p_{1}, p_{2}\right)$ at the symmetric equilibrium, we find:

$$
\frac{\partial p_{1}}{\partial \mu_{1}} \frac{1}{2}+\frac{\partial\left(p_{2}-p_{1}\right)}{\partial \mu_{1}} \frac{1}{2 t}\left(1+\delta \mu_{1}+p_{1}\right)
$$

where $\frac{\partial\left(p_{2}-p_{1}\right)}{\partial \mu_{1}}<0$ if and only if $2 \Delta u>\delta$. In particular, $t \leq \frac{4}{3}$ and $2 \Delta u>\delta$ imply that the sum of the two terms in (17) has a negative sign. Basically, if 1's quality increases, 1 can appropriate it by increasing its price $p_{1}$ but the price gap $p_{2}-p_{1}$ decreases, which reduces 1 's market share. When competition is strong enough, the second effect dominates the first effect such that paywalls soften quality competition.

\section{Conclusion}

In this paper, we studied the impact of a news aggregator on the quality choices of newspapers by considering two scenarios: symmetric newspapers and asymmetric newspapers. In both scenarios we find that the presence of the news aggregator induces each newspaper to specialize, in order to 
boost its traffic from the aggregator. When newspapers are symmetric, the aggregator induces them to choose higher quality, which increases consumer surplus and social welfare. When newspapers are asymmetric such that small newspapers prefer their content to be indexed by the aggregator, the aggregator can increase or decrease the quality chosen by large newspapers depending on how sensitively time spent on news sites responds to quality increase. We found that if adding content indexed by the aggregator increases the traffic to all the newspapers that have been indexed by the aggregator (as in the empirical findings of Athey and Mobius (2012) and Chiou and Tucker (2012)), then the aggregator increases the quality (and thereby consumer surplus and welfare).

Our model is a first step to study the role of news aggregators, opening many avenues for future research. We described consumer behavior in a highly-stylized way. It would be nice to enrich consumer behavior by enabling multi-homing without aggregator (Kim and Serfes, 2006), allowing each consumer to read a subset of articles depending on her types or introducing richer interactions between utility from reading and ideological slant. It would be also interesting to analyze the (long-term) impact of the aggregators on news slanting by making each newspaper's position endogenous, as in Gabszewicz, Laussel, and Sonnac (2001) and Mullainathan and Shleifer (2005).

\section{References}

Anderson, S. P., And S. Coate (2005): "Market Provision of Broadcasting: A Welfare Analysis," The Review of Economic Studies, 72(4), pp. 947-972.

Anderson, S. P., Ø. Foros, H. J. Kind, And M. Peitz (2012): "Media market concentration, advertising levels, and ad prices," International Journal of Industrial Organization, 30(3), 321 -325 .

Anderson, S. P., And J. S. Gans (2011): "Platform Siphoning: Ad-Avoidance and Media Content," American Economic Journal: Microeconomics, 3(4), pp. 1-34.

Armstrong, M. (2006): "Competition in Two-Sided Markets," The RAND Journal of Economics, 37(3), pp. 668-691.

Athey, S., E. Calvano, and J. S. Gans (2012): "The Impact of the Internet on Advertising Markets for News Media," Working paper.

Athey, S., And M. Mobius (2012): "The Impact of News Aggregators on Internet News Consumption: The Case of Localization," Working paper. 
Baye, M. R., and J. Morgan (2001): "Information Gatekeepers on the Internet and the Competitiveness of Homogeneous Product Markets," The American Economic Review, 91(3), pp. $454-474$.

Biglaiser, G. (1993): "Middlemen as Experts," The RAND Journal of Economics, 24(2), pp. $212-223$.

Caillaud, B., and B. Jullien (2001): "Competing cybermediaries," European Economic Review, 45(4-6), 797-808.

(2003): "Chicken and Egg: Competition among Intermediation Service Providers," The RAND Journal of Economics, 34(2), pp. 309-328.

CAlzada, J., And G. ORdóÑEz (2012): "Competition in the news industry: fighting aggregators with versions and links," Working Papers 12-22, NET Institute.

Chiou, L., And C. Tucker (2012): "Copyright, Digitization, and Aggregation," Discussion paper, NET Institute Working Paper No. 11-18.

Crampes, C., C. Haritchabalet, and B. Jullien (2009): "Advertising, Competition and Entry in Media Industries," The Journal of Industrial Economics, 57(1), pp. 7-31.

Crémer, J., P. Rey, and J. Tirole (2000): "Connectivity in the Commercial Internet," The Journal of Industrial Economics, 48(4), pp. 433-472.

Dellarocas, C., Z. Katona, and W. Rand (2012): "Media, Aggregators and the Link Economy: Strategic Hyperlink Formation in Content Networks," Working Papers 10-13, NET Institute.

Farrell, J., and G. Saloner (1985): "Standardization, Compatibility, and Innovation," The RAND Journal of Economics, 16(1), pp. 70-83.

(1986): "Installed Base and Compatibility: Innovation, Product Preannouncements, and Predation," The American Economic Review, 76(5), pp. 940-955.

FTC (2010): "Potential Policy Recommendations To Support The Reinvention Of Journalism," Discussion draft, Federal Trade Commission.

Gabszewicz, J. J., D. Laussel, and N. Sonnac (2001): "Press advertising and the ascent of the 'Pensée Unique'," European Economic Review, 45(4-6), 641 - 651.

Gentzkow, M., and J. M. Shapiro (2011): "Ideological Segregation Online and Offline," The Quarterly Journal of Economics, 126(4), 1799-1839. 
George, L., And C. Hogendorn (2012): "Aggregators, search and the economics of new media institutions," Information Economics and Policy, 24(1), 40 - 51.

(2013): "Local News Online: Aggregators, Geo-Targeting and the Market for Local News," Working paper.

Google (2010): "Comments on Federal Trade Commission's News Media Workshop and Staff Discussion Draft on "Potential Policy Recommendations to Support the Reinvention of Journalism"," Discussion paper.

Hagiu, A. (2006): "Pricing and Commitment by Two-Sided Platforms," The RAND Journal of Economics, 37(3), pp. 720-737.

Hotelling, H. (1929): "Stability in Competition," The Economic Journal, 39(153), pp. 41-57.

Jeon, D.-S., And D. MeniCuCCi (2011): "Interconnection among academic journal websites: multilateral versus bilateral interconnection," The RAND Journal of Economics, 42(2), 363386.

Katz, M. L., And C. Shapiro (1985): "Network Externalities, Competition, and Compatibility," The American Economic Review, 75(3), pp. 424-440.

Kim, H., And K. Serfes (2006): "A Location Model with Preference for Variety," The Journal of Industrial Economics, 54(4), pp. 569-595.

LizzerI, A. (1999): "Information Revelation and Certification Intermediaries," The RAND Journal of Economics, 30(2), pp. 214-231.

Mullainathan, S., And A. Shleifer (2005): "The Market for News," The American Economic Review, 95(4), pp. 1031-1053.

Murdoch, R. (2009): "From Town Crier to Bloggers: How Will Journalism Survive the Internet Age?," Before the federal trade commission's workshop.

Outsell (2009): "News Users," Discussion paper.

Parker, G. G., and M. W. Van Alstyne (2005): "Two-Sided Network Effects: A Theory of Information Product Design," Manage. Sci., 51(10), 1494-1504.

Peitz, M., And T. M. Valletti (2008): "Content and advertising in the media: Pay-tv versus free-to-air," International Journal of Industrial Organization, 26(4), 949 - 965. 
Pew Research Center (2012): "Trends in News Consumption: 1991-2012: In Changing News Landscape, Even Television is Vulnerable," Discussion paper, Pew Research Center For The People and The Press.

Rochet, J.-C., And D.-S. Jeon (2010): "The Pricing of Academic Journals: A Two-Sided Market Perspective," American Economic Journal: Microeconomics, 2(2), 222-55.

Rochet, J.-C., And J. Tirole (2002): "Cooperation among Competitors: Some Economics of Payment Card Associations," The RAND Journal of Economics, 33(4), pp. 549-570.

(2003): "Platform Competition in Two-sided Markets," Journal of the European Economic Association, 1(4), pp. 990-1029.

(2006): "Two-Sided Markets: A Progress Report," The RAND Journal of Economics, 37(3), pp. 645-667.

Rutt, J. (2011): "Aggregators and the News Industry: Charging for Access to Content," Working Papers 11-19, NET Institute.

The State of the News Media (2013): "Project for Excellence in Journalism," Discussion paper, Pew Research Center.

Tirole, J. (1988): The theory of industrial organization. Massachusetts Institute of Technology.

Weyl, E. G. (2010): "A Price Theory of Multi-sided Platforms," American Economic Review, $100(4), 1642-72$. 


\section{Appendix A}

\subsection{Proof of Lemma 3}

Proof. We prove it for newspaper 1. $U^{1}\left(x_{1}\right)=U^{A g g}\left(x_{1}\right)$ is equivalent to

$$
x_{1}=\frac{1}{2}-\beta \frac{\mu\left(s_{1} \cup s_{2}\right)-\mu\left(s_{1}\right)}{1-\mu\left(s_{1}\right)+\mu\left(s_{2}\right)} .
$$

Using $\mu\left(s_{1} \cup s_{2}\right)=\mu\left(s_{1}\right)+\mu\left(s_{2}\right)-\mu\left(s_{1} \cap s_{2}\right)$, we get

$$
x_{1}=\frac{1}{2}-\beta \frac{\mu\left(s_{2}\right)-\mu\left(s_{1} \cap s_{2}\right)}{1-\mu\left(s_{1}\right)+\mu\left(s_{2}\right)} .
$$

We now show $0<x_{1} \leq 1 / 2$, which is equivalent to

$$
0 \leq \beta \frac{\mu\left(s_{2}\right)-\mu\left(s_{1} \cap s_{2}\right)}{1-\mu\left(s_{1}\right)+\mu\left(s_{2}\right)}<\frac{1}{2}
$$

The first inequality is straightforward. The second comes from

$$
\beta \frac{\mu\left(s_{2}\right)-\mu\left(s_{1} \cap s_{2}\right)}{1-\mu\left(s_{1}\right)+\mu\left(s_{2}\right)}<\frac{\mu\left(s_{2}\right)}{1-\mu\left(s_{1}\right)+\mu\left(s_{2}\right)} \leq \frac{\mu\left(s_{2}\right)}{1 / 2+\mu\left(s_{2}\right)}<\frac{1}{2} .
$$

\subsection{Proof of Proposition 1}

Proof. There are four equilibrium candidates.

1) $\left(\frac{1}{2}, \frac{1}{2}\right)$ : This is an equilibrium if and only if $\frac{1}{2} \leq 1-\frac{2 c-\delta-\beta}{\delta \beta}$, or equivalently $c \leq \frac{\delta \beta}{4}+\frac{\delta}{2}+\frac{\beta}{2}$.

$2 \& 3)\left(\frac{1}{2}, \frac{\delta+\beta-\frac{\delta \beta}{2}}{4 c-2 \delta \beta}\right)$ and $\left(\frac{\delta+\beta-\frac{\delta \beta}{2}}{4 c-2 \delta \beta}, \frac{1}{2}\right)$ : To have an equilibrium of this kind, the following conditions should hold

- $\frac{\delta+\beta-\frac{\delta \beta}{2}}{4 c-2 \delta \beta} \leq 1-\frac{2 c-\delta-\beta}{\delta \beta} \Leftrightarrow\left(c-\frac{\delta}{2}-\frac{\beta}{2}-\frac{\delta \beta}{4}\right)\left(c-\frac{3}{4} \delta \beta\right) \leq 0$

- $1-\frac{2 c-\delta-\beta}{\delta \beta}<\frac{1}{2} \Leftrightarrow c>\frac{\delta}{2}+\frac{\beta}{2}+\frac{\delta \beta}{4} \Rightarrow c>\frac{3}{4} \delta \beta$.

As these conditions can not satisfy simultaneously, there exists no equilibrium of this kind.

4) $\left(\frac{\delta+\beta}{4 c-\delta \beta}, \frac{\delta+\beta}{4 c-\delta \beta}\right)$ : This is an equilibrium if and only if $\frac{1}{2}>\frac{\delta+\beta}{4 c-\delta \beta}>1-\frac{2 c-\delta-\beta}{\delta \beta}$, or equivalently $c>\frac{\delta \beta}{4}+\frac{\delta}{2}+\frac{\beta}{2}$. 


\subsection{Proof of Proposition 2}

Proof. We prove the proposition for newspaper 1. We decompose the profit of the newspaper 1, (8), using (6), (7), $\mu\left(s_{1} \cup s_{2}\right)=\mu\left(s_{1}\right)+\mu\left(s_{2}\right)-\mu\left(s_{1} \cap s_{2}\right), \mu\left(s_{1}-s_{2}\right)=\mu\left(s_{1}\right)-\mu\left(s_{1} \cap s_{2}\right)$ and $\mu\left(s_{2}-s_{1}\right)=\mu\left(s_{2}\right)-\mu\left(s_{1} \cap s_{2}\right)$.

$$
\begin{aligned}
\pi_{1}\left(s_{1}\right) & =\delta \alpha_{1} \mu\left(s_{1}\right)+\alpha_{1}+\delta\left(1-\alpha_{1}-\alpha_{2}\right)\left(\mu\left(s_{1}\right)-\frac{1}{2} \mu\left(s_{1} \cap s_{2}\right)\right)-c \mu\left(s_{1}\right)^{2} \\
& =h\left(\mu\left(s_{1}\right), \mu\left(s_{2}\right)\right)+\frac{\delta \beta \mu\left(s_{1} \cap s_{2}\right)}{1-\left(\mu\left(s_{1}\right)-\mu\left(s_{2}\right)\right)^{2}}\left[\mu\left(s_{1} \cap s_{2}\right)-g\left(\mu\left(s_{1}\right), \mu\left(s_{2}\right)\right)\right],
\end{aligned}
$$

where

$$
\begin{aligned}
& h\left(\mu\left(s_{1}\right), \mu\left(s_{2}\right)\right)=\frac{1}{2}+\frac{\delta}{2} \mu\left(s_{1}\right)-\beta \frac{\mu\left(s_{2}\right)}{1+\mu\left(s_{2}\right)-\mu\left(s_{1}\right)}+\delta \beta \frac{\mu\left(s_{1}\right)^{2}}{1+\mu\left(s_{1}\right)-\mu\left(s_{2}\right)}-c \mu\left(s_{1}\right)^{2} \\
& g\left(\mu\left(s_{1}\right), \mu\left(s_{2}\right)\right)=-\frac{3}{2} \mu\left(s_{1}\right)^{2}+\mu\left(s_{1}\right)\left(2 \mu\left(s_{2}\right)-\frac{1}{\delta}+\frac{3}{2}\right)+\left(1-\mu\left(s_{2}\right)\right)\left(\frac{1}{2} \mu\left(s_{2}\right)-\frac{1}{\delta}\right)
\end{aligned}
$$

The profit function is convex with respect to $\mu\left(s_{1} \cap s_{2}\right), \frac{\partial^{2} \pi_{1}\left(s_{1}\right)}{\partial \mu\left(s_{1} \cap s_{2}\right)^{2}}=\frac{2 \delta \beta}{1-\left(\mu\left(s_{1}\right)-\mu\left(s_{2}\right)\right)^{2}}>0$. Therefore, it is maximized at the corners, $\mu\left(s_{1} \cap s_{2}\right)=0$ or $\mu\left(s_{1} \cap s_{2}\right)=\min \left(\mu\left(s_{1}\right), \mu\left(s_{2}\right)\right)$.

\subsection{Proof of Proposition 3}

Proof. We do the proof in two steps. First we compute symmetric equilibria, given minimum differentiation, $\mu\left(s_{1} \cap s_{2}\right)=\min \left(\mu\left(s_{1}\right), \mu\left(s_{2}\right)\right)$. Second, we find under which condition minimum differentiation equilibria exist.

1) Under minimum differentiation, the newspaper 1's profit and its derivative is:

$$
\begin{gathered}
\pi_{1}\left(s_{1} \mid \min \right)= \begin{cases}\frac{\delta}{2} \mu_{1}+\frac{1}{2}+\delta \beta \frac{\left(\mu_{1}-\mu_{2}\right)\left(\mu_{1}-\frac{1}{2} \mu_{2}\right)}{1+\mu_{1}-\mu_{2}}-c \mu_{1}^{2} & \mu_{1}>\mu_{2} \\
\frac{\delta}{2} \mu_{1}+\frac{1}{2}-\frac{\delta \beta}{2} \frac{\left(\mu_{2}-\mu_{1}\right) \mu_{1}}{1+\mu_{2}-\mu_{1}}-\beta \frac{\left(\mu_{2}-\mu_{1}\right)}{1+\mu_{2}-\mu_{1}}-c \mu_{1}^{2} & \mu_{1} \leq \mu_{2}\end{cases} \\
\pi_{1}^{\prime}\left(s_{1} \mid \min \right)= \begin{cases}\frac{\delta}{2}+\delta \beta \frac{\left(\mu_{1}-\mu_{2}\right)}{1+\mu_{1}-\mu_{2}}+\delta \beta \frac{\left(\mu_{1}-\frac{1}{2} \mu_{2}\right)}{\left(1+\mu_{1}-\mu_{2}\right)^{2}}-2 c \mu_{1} & \mu_{1}>\mu_{2} \\
\frac{\delta}{2}-\frac{\delta \beta}{2} \frac{\left(\mu_{2}-\mu_{1}\right)}{1+\mu_{2}-\mu_{1}}+\frac{\delta \beta}{2} \frac{\mu_{1}}{\left(1+\mu_{2}-\mu_{1}\right)^{2}}+\frac{\beta}{\left(1+\mu_{2}-\mu_{1}\right)^{2}}-2 c \mu_{1} & \mu_{1}<\mu_{2}\end{cases}
\end{gathered}
$$

Any symmetric equilibrium candidate, $(\mu, \mu)$, can be seen in three cases: 
1.1) $\mu<\frac{\delta}{4 c-\delta \beta}$ : In this case, there is always a deviation.

$$
\pi_{1}^{\prime}\left(s_{1} \mid \min , \mu_{1}=\mu_{2}=\mu\right)^{+}=\frac{\delta}{2}+\frac{\delta \beta}{2} \mu-2 c \mu=\frac{4 c-\delta \beta}{2}\left(\frac{\delta}{4 c-\delta \beta}-\mu\right)>0
$$

Thus, newspaper 1 benefits from investing more on quality.

1.2) $\frac{\delta}{4 c-\delta \beta} \leq \mu<\min \left\{\frac{\delta+2 \beta}{4 c-\delta \beta}, \frac{1}{2}\right\}$ : We show for any $\frac{\delta}{4 c-\delta \beta} \leq \mu_{2}<\min \left\{\frac{\delta+2 \beta}{4 c-\delta \beta}, \frac{1}{2}\right\}$ the best response of newspaper 1 is $\mu_{2}, B R_{1}\left(\mu_{2}\right)=\mu_{2}$.

$$
\begin{aligned}
\pi_{1}\left(s_{1} \mid \min , \mu_{1} \geq \mu_{2}\right) & =\frac{\delta}{2} \mu_{1}+\frac{1}{2}+\delta \beta \frac{\left(\mu_{1}-\mu_{2}\right)\left(\mu_{1}-\frac{1}{2} \mu_{2}\right)}{1+\mu_{1}-\mu_{2}}-c \mu_{1}^{2} \\
& =\frac{1}{2}+\frac{\delta}{2} \mu_{2}-c \mu_{2}^{2}+\left(\mu_{1}-\mu_{2}\right) k\left(\mu_{1}, \mu_{2}\right) \\
& =\pi_{1}\left(s_{1} \mid \min , \mu_{1}=\mu_{2}\right)+\left(\mu_{1}-\mu_{2}\right) k\left(\mu_{1}, \mu_{2}\right)
\end{aligned}
$$

where

$$
\begin{aligned}
k\left(\mu_{1}, \mu_{2}\right) & =\frac{\delta}{2}+\delta \beta \frac{\left(\mu_{1}-\frac{1}{2} \mu_{2}\right)}{1+\mu_{1}-\mu_{2}}-c\left(\mu_{1}+\mu_{2}\right) \\
& =\frac{\delta}{2}+\delta \beta \frac{\left(\mu_{1}-\frac{1}{2} \mu_{2}\right)}{1+\mu_{1}-\mu_{2}}-c\left(\mu_{1}-\mu_{2}\right)-2 c \mu_{2} \\
& \leq \frac{\delta}{2}+\delta \beta \frac{\left(\mu_{1}-\frac{1}{2} \mu_{2}\right)}{1+\mu_{1}-\mu_{2}}-c\left(\mu_{1}-\mu_{2}\right)-\frac{\delta}{2}-\frac{\delta \beta}{2} \mu_{2} \\
& =\left(\mu_{1}-\mu_{2}\right)\left(\delta \beta \frac{\left(1-\frac{1}{2} \mu_{2}\right)}{1+\mu_{1}-\mu_{2}}-c\right) \\
& \leq\left(\mu_{1}-\mu_{2}\right)\left(\delta \beta\left(1-\frac{1}{2} \mu_{2}\right)-c\right) \\
& \leq\left(\mu_{1}-\mu_{2}\right)\left(\delta \beta\left(1-\frac{1}{2} \frac{\delta}{4 c-\delta \beta}\right)-c\right) \\
& \leq \frac{\left(\mu_{1}-\mu_{2}\right)}{4 c-\delta \beta}\left(-4 c^{2}+5 \delta \beta c-\delta \beta\left(\delta \beta+\frac{\delta}{2}\right)\right) \\
\leq & \frac{\left(\mu_{1}-\mu_{2}\right)}{4 c-\delta \beta}\left(-4 c^{2}+5 \delta \beta c-\delta \beta\left(\delta \beta+\frac{\delta \beta}{2}\right)\right) \\
& \frac{\left(\mu_{1}-\mu_{2}\right)}{4 c-\delta \beta}(-4)\left(c-\frac{3}{4} \delta \beta\right)\left(c-\frac{\delta \beta}{2}\right)<0
\end{aligned}
$$


Therefore, $\pi_{1}\left(s_{1} \mid \min , \mu_{1}=\mu_{2}\right)>\pi_{1}\left(s_{1} \mid \min , \mu_{1}>\mu_{2}\right)$.

$$
\begin{aligned}
\pi_{1}\left(s_{1} \mid \min , \mu_{1} \leq \mu_{2}\right) & =\frac{\delta}{2} \mu_{1}+\frac{1}{2}-\frac{\delta \beta}{2} \frac{\left(\mu_{2}-\mu_{1}\right) \mu_{1}}{1+\mu_{2}-\mu_{1}}-\beta \frac{\left(\mu_{2}-\mu_{1}\right)}{1+\mu_{2}-\mu_{1}}-c \mu_{1}^{2} \\
& =\frac{1}{2}+\frac{\delta}{2} \mu_{2}-c \mu_{2}^{2}+\left(\mu_{1}-\mu_{2}\right) z\left(\mu_{1}, \mu_{2}\right) \\
& =\pi_{1}\left(s_{1} \mid \min , \mu_{1}=\mu_{2}\right)+\left(\mu_{1}-\mu_{2}\right) z\left(\mu_{1}, \mu_{2}\right),
\end{aligned}
$$

where

$$
z\left(\mu_{1}, \mu_{2}\right)=\frac{\delta}{2}+\frac{\delta \beta}{2} \frac{\mu_{1}}{1+\mu_{2}-\mu_{1}}+\frac{\beta}{1+\mu_{2}-\mu_{1}}-c\left(\mu_{1}+\mu_{2}\right) .
$$

The derivative of $z$ with respect to $\mu_{1}$ is

$$
\frac{\partial z\left(\mu_{1}, \mu_{2}\right)}{\partial \mu_{1}}=\frac{\delta \beta}{2} \frac{1+\mu_{2}}{\left(1+\mu_{2}-\mu_{1}\right)^{2}}+\frac{\beta}{\left(1+\mu_{2}-\mu_{1}\right)^{2}}-c
$$

Since $z$ is convex with respect to $\mu_{1}$, it achieves its minimum at $\underline{\mu}$, where $\frac{\partial z\left(\mu, \mu_{2}\right)}{\partial \mu_{1}}=0$, or equivalently:

$$
\frac{\beta}{1+\mu_{2}-\underline{\mu}}=-\frac{\delta \beta}{2} \frac{1+\mu_{2}}{1+\mu_{2}-\underline{\mu}}+c\left(1+\mu_{2}-\underline{\mu}\right) .
$$

Hence,

$$
\begin{aligned}
z\left(\mu_{1}, \mu_{2}\right) & \geq z\left(\underline{\mu}, \mu_{2}\right) \\
& =\frac{\delta}{2}+\frac{\delta \beta}{2} \frac{\underline{\mu}}{1+\mu_{2}-\underline{\mu}}+\frac{\beta}{1+\mu_{2}-\underline{\mu}}-c\left(\underline{\mu}+\mu_{2}\right) \\
& =\frac{\delta}{2}+\frac{\delta \beta}{2} \frac{\underline{\mu}}{1+\mu_{2}-\underline{\mu}}-\frac{\delta \beta}{2} \frac{1+\mu_{2}}{1+\mu_{2}-\underline{\mu}}+c\left(1+\mu_{2}-\underline{\mu}\right)-c\left(\underline{\mu}+\mu_{2}\right) \\
& =\frac{\delta}{2}-\frac{\delta \beta}{2}+c(1-2 \underline{\mu})>0 .
\end{aligned}
$$

As a result, $\pi_{1}\left(s_{1} \mid \min , \mu_{1}=\mu_{2}\right)>\pi_{1}\left(s_{1} \mid \min , \mu_{1}<\mu_{2}\right)$. We can conclude $B R_{1}\left(\mu_{2}\right)=\mu_{2}$. Therefore, any $(\mu, \mu)$, such that $\mu \in\left[\frac{\delta}{4 c-\delta \beta}, \min \left\{\frac{\delta+2 \beta}{4 c-\delta \beta}, \frac{1}{2}\right\}\right]$, is an equilibrium under minimum differentiation.

1.3) $\mu>\min \left\{\frac{\delta+2 \beta}{4 c-\delta \beta}, \frac{1}{2}\right\}$ : Thanks to A2, we only focus on the case where $\frac{\delta+2 \beta}{4 c-\delta \beta}<\mu<\frac{1}{2}$. There is always a deviation in this case.

$$
\pi_{1}^{\prime}\left(s_{1} \mid \min , \mu_{1}=\mu_{2}=\mu\right)^{-}=\frac{\delta}{2}+\frac{\delta \beta}{2} \mu+\beta-2 c \mu=\frac{4 c-\delta \beta}{2}\left(\frac{\delta+2 \beta}{4 c-\delta \beta}-\mu\right)<0
$$

Therefore, newspaper 1 benefits from reducing its investment on quality. 
2) So far, we pin down all symmetric equilibria under minimum differentiation. This means there is no deviation given $\mu\left(s_{1} \cap s_{2}\right)=\min \left(\mu\left(s_{1}\right), \mu\left(s_{2}\right)\right)$. However, we should check for any deviations with $\mu\left(s_{1} \cap s_{2}\right)<\min \left\{\mu\left(s_{1}\right), \mu\left(s_{2}\right)\right\}$. According to Proposition 2, the most profitable deviation for newspaper 1 is $s_{1} \cap s_{2}=\emptyset$. For any equilibrium candidate $(\mu, \mu)$, the maximum gain of newspaper 1 from deviation to $\mu_{1}$ is:

$$
\begin{aligned}
d\left(\mu_{1}, \mu, \delta, \beta, c\right)= & \frac{\delta}{2} \mu+\frac{1}{2}-c \mu^{2}-\left(\delta \alpha_{1} \mu_{1}+\alpha_{1}+\delta\left(1-\alpha_{1}-\alpha_{2}\right) \mu_{1}-c \mu_{1}^{2}\right) \\
= & c \mu_{1}^{4}-\left(\frac{\delta}{2}+\delta \beta+2 c \mu\right) \mu_{1}^{3}+\left(\frac{3 \delta}{2} \mu+\delta \beta(1+\mu)-c\right) \mu_{1}^{2} \\
& +\left(\frac{\delta}{2}-\frac{3 \delta}{2} \mu^{2}-\beta \mu+2 c \mu^{3}\right) \mu_{1}-\frac{\delta}{2} \mu+\frac{\delta}{2} \mu^{3}-\beta \mu+\beta \mu^{2}+c \mu^{2}-c \mu^{4} .
\end{aligned}
$$

Approaching $\delta$ to zero, $\delta \rightarrow 0$, implies $\mu<\frac{\beta}{2 c}$. Therefore,

$$
\begin{aligned}
\lim _{\delta \rightarrow 0} d\left(\mu_{1}, \mu, \delta, \beta, c\right) & =c \mu_{1}^{4}-(2 c \mu) \mu_{1}^{3}+(-c) \mu_{1}^{2}+\left(-\beta \mu+2 c \mu^{3}\right) \mu_{1}-\beta \mu+\beta \mu^{2}+c \mu^{2}-c \mu^{4} \\
& <c \mu_{1}^{4}-(2 c \mu) \mu_{1}^{3}+(-c) \mu_{1}^{2}+\left(-\beta \mu+\beta \mu^{2}\right) \mu_{1}-\beta \mu+\beta \mu^{2}+\frac{\beta}{2} \mu-c \mu^{4} \\
& =-c \mu_{1}^{2}\left(1-\mu_{1}^{2}\right)-(2 c \mu) \mu_{1}^{3}-\beta \mu(1-\mu) \mu_{1}-\beta \mu\left(\frac{1}{2}-\mu\right)-c \mu^{4}<0 .
\end{aligned}
$$

This implies that there exists a $\underline{\delta}^{m}>0$, such that for all $\delta \leq \underline{\delta}^{m}$ the gain from deviation is negative, $d\left(\mu_{1}, \mu, \delta, \beta, c\right)<0$, due to continuity of $d$. This means $(\mu, \mu)$ is an equilibrium.

$(\mu, \mu)$ can not be sustained as an equilibrium, if $d(\mu, \mu, \delta, \beta, c)>0$.

$$
\begin{aligned}
d(\mu, \mu, \delta, \beta, c) & =\delta \beta \mu^{2}-\beta \mu>0 \Leftrightarrow \delta \mu>1 \\
& \Rightarrow \delta\left(\frac{\delta+2 \beta}{4 c-\delta \beta}\right)>1 \\
& \Rightarrow \delta^{2}+3 \delta \beta-4 c>0 \Rightarrow \delta>\bar{\delta}^{m}
\end{aligned}
$$

where $\bar{\delta}^{m}$ is the positive solution of $\delta^{2}+3 \delta \beta-4 c$. Therefore, for all $\delta>\bar{\delta}^{m}$ there exists no minimum differentiation equilibrium.

\subsection{Proof of Proposition 4}

Proof. First, we compute the equilibrium candidate, and then we find the condition under which the equilibrium sustained.

Under maximum differentiation, $\mu\left(s_{1} \cap s_{2}\right)=0$, the newspaper 1's profit and its derivatives are: 


$$
\begin{gathered}
\pi_{1}\left(s_{1} \mid \max \right)=\frac{1}{2}+\frac{\delta}{2} \mu_{1}-\beta \frac{\mu_{2}}{1+\mu_{2}-\mu_{1}}+\delta \beta \frac{\mu_{1}^{2}}{1+\mu_{1}-\mu_{2}}-c \mu_{1}^{2} \\
\pi_{1}^{\prime}\left(s_{1} \mid \max \right)=\frac{\delta}{2}-\beta \frac{\mu_{2}}{\left(1+\mu_{2}-\mu_{1}\right)^{2}}+2 \delta \beta \frac{\mu_{1}}{1+\mu_{1}-\mu_{2}}-\delta \beta \frac{\mu_{1}^{2}}{\left(1+\mu_{1}-\mu_{2}\right)^{2}}-2 c \mu_{1} \\
\pi_{1}^{\prime \prime}\left(s_{1} \mid \max \right)=-2 \beta \frac{\mu_{2}}{\left(1+\mu_{2}-\mu_{1}\right)^{3}}+\frac{2 \delta \beta\left(1-\mu_{2}\right)^{2}}{\left(1+\mu_{1}-\mu_{2}\right)^{3}}-2 c \\
\pi_{1}^{\prime \prime \prime}\left(s_{1} \mid \max \right)=-6 \beta \frac{\mu_{2}}{\left(1+\mu_{2}-\mu_{1}\right)^{4}}-\frac{6 \delta \beta\left(1-\mu_{2}\right)^{2}}{\left(1+\mu_{1}-\mu_{2}\right)^{4}}
\end{gathered}
$$

From Lemma 4 , we know $\delta>2$ is a necessary condition. Therefore,

$$
\pi_{1}^{\prime}\left(s_{1} \mid \max , \mu_{1}=0\right)=\frac{\delta}{2}-\beta \frac{\mu_{2}}{\left(1+\mu_{2}\right)^{2}} \geq \frac{\delta}{2}-\beta \frac{2}{9}>0 .
$$

This and the negativity of $\pi_{1}^{\prime \prime \prime}$ imply that $\pi_{1}$ achieves its maximum at either $\frac{1}{2}$ or the solution of $\pi_{1}^{\prime}\left(s_{1} \mid \max \right)=0$. As we are looking for symmetric equilibrium, there are not more than two possibilities:

1) $\left(\frac{1}{2}, \frac{1}{2}\right)$ is an equilibrium, if $\pi_{1}^{\prime}\left(s_{1} \mid \max , \mu_{1}=\mu_{2}=\frac{1}{2}\right)>0$, or equivalently $c \leq \frac{\delta}{2}-\frac{\beta}{2}+\frac{3}{4} \delta \beta$.

2) $(\hat{\mu}, \hat{\mu})$, where $\hat{\mu}$ is the solution of

$$
Q(\hat{\mu})=\hat{\mu}^{2}(-\delta \beta)+\hat{\mu}(-\beta+2 \delta \beta-2 c)+\frac{\delta}{2}=0,
$$

which is obtained from putting $\mu_{1}=\mu_{2}=\hat{\mu}$ in (21). And $\hat{\mu}<\frac{1}{2}$ if and only if $c>\frac{\delta}{2}-\frac{\beta}{2}+\frac{3}{4} \delta \beta$.

So far we have shown that there is no deviation given the maximum differentiation. However, we should check for any deviation with $\mu\left(s_{1} \cap s_{2}\right)>0$. According to the Proposition 2 , the most profitable deviation is choosing the minimum differentiation, $\mu\left(s_{1} \cap s_{2}\right)=\min \left(\mu\left(s_{1}\right), \mu\left(s_{2}\right)\right)$.

Suppose $(\mu, \mu)$ is the equilibrium candidate. We consider two deviations:

a) $\mu_{1} \leq \mu$ : There is no profitable deviation, if:

$$
\frac{1}{2}+\frac{\delta}{2} \mu-\beta \mu+\delta \beta \mu^{2}-c \mu^{2} \geq \frac{1}{2}+\frac{\delta}{2} \mu_{1}-\frac{\delta \beta}{2} \frac{\left(\mu-\mu_{1}\right) \mu_{1}}{1+\mu-\mu_{1}}-\beta \frac{\mu-\mu_{1}}{1+\mu-\mu_{1}}-c \mu_{1}^{2},
$$

where the LHS represent the profit in equilibrium, $(\mu, \mu)$, and RHS shows the profit of newspaper 1 when she deviates from the equilibrium, $(\mu, \mu)$, to $\left(\mu_{1}, \mu\right)$ with minimum differentiation. The inequality is equivalent to 


$$
\left(\mu-\mu_{1}\right)\left(\delta\left(-\frac{1}{2}-\frac{\beta}{2} \frac{\mu_{1}}{1+\mu-\mu_{1}}\right)+c\left(\mu+\mu_{1}\right)-\beta \frac{1}{1+\mu-\mu_{1}}\right)+\beta \mu-\delta \beta \mu^{2} \leq 0 .
$$

As the coefficient of $\delta$ in the inequality is negative, there exist a $\bar{\delta}_{1}>0$ such that for all $\delta>\bar{\delta}_{1}$ the left term takes negative values. The negativity of right term, i.e. $\delta \mu>1$, is a necessary condition to have maximum differentiation equilibrium (Lemma 4). Because newspaper 1 can deviate by keeping the quality the same, $\mu_{1}=\mu$, but choosing minimum differentiation. $\delta \mu>1$ if and only if:

$$
Q\left(\frac{1}{\delta}\right)>0 \Leftrightarrow \delta^{2}+4 \delta \beta-4 c-4 \beta>0 \Leftrightarrow \delta>\bar{\delta}_{2},
$$

where $\bar{\delta}_{2}$ is the positive solution of $\delta^{2}+4 \delta \beta-4 c-4 \beta$.

b) $\mu_{1} \geq \mu$ : In this case $\mu\left(s_{1} \cap s_{2}\right)=\mu$. According to the proof of Proposition 2, this deviation is profitable if $\mu>g\left(\mu_{1}, \mu\right)$. From (20), we know $\frac{\partial g\left(\mu_{1}, \mu\right)}{\partial \mu_{1}}=3\left(\frac{1}{2}-\mu_{1}\right)+2 \mu-\frac{1}{\delta}>0$, and $g(\mu, \mu)=2 \mu-\frac{1}{\delta}>\mu$. Therefore, $\mu<g\left(\mu_{1}, \mu\right)$ for all $\mu_{1} \geq \mu$. This means there is no profitable deviation, such that $\mu_{1}>\mu$.

Therefore, for all $\delta>\bar{\delta}^{M}=\max \left\{\bar{\delta}_{1}, \bar{\delta}_{2}\right\}$ there exists an equilibrium in which newspapers invest on different sets of issues.

Moreover, we can set $\underline{\delta}^{M}=\bar{\delta}_{2}$ which implies for all $\delta<\underline{\delta}^{M}$ there exists no maximum differentiation equilibrium.

\subsection{Proof of Proposition 6}

Proof. In terms of $c$, we have two cases:

1) $c>\frac{\delta}{2}-\frac{\beta}{2}+\frac{3}{4} \delta \beta$ : From (22), we have

$$
Q\left(\mu^{M}\right)=\mu^{M^{2}}(-\delta \beta)+\mu^{M}(-\beta+2 \delta \beta-2 c)+\frac{\delta}{2}=0
$$

From Proposition 1, we know $2 c \mu^{*}-\frac{\beta}{2}-\frac{\delta \beta}{2} \mu^{*}-\frac{\delta}{2}=0$. By adding this to the $Q\left(\mu^{M}\right)$, we get

$$
\begin{aligned}
2\left(\mu^{M}-\mu^{*}\right)\left(c-\frac{\delta \beta}{4}\right) & =\mu^{M^{2}}(-\delta \beta)+\left(\frac{3 \delta \beta}{2}-\beta\right) \mu^{M}-\frac{\beta}{2} \\
& =\delta \beta \mu^{M}\left(\frac{1}{2}-\mu^{M}\right)+\beta \mu^{M}\left(\frac{\delta}{2}-1\right)+\frac{\beta}{2}\left(\mu^{M} \delta-1\right) \\
& \geq 0,
\end{aligned}
$$

where we have $\mu^{M} \delta>1$ from Lemma 4 to prove the inequality. Hence, $\mu^{M} \geq \mu^{*}$.

2) $c \leq \frac{\delta}{2}-\frac{\beta}{2}+\frac{3}{4} \delta \beta$ : In this case, $\mu^{M}=\frac{1}{2}$, and therefore $\mu^{M} \geq \mu^{*}$. 


\subsection{Proof of Proposition 7}

Proof. (ii) First, we show $\pi^{M}-\pi^{*}$ is decreasing with $c$. We consider two cases: a) $c \geq \frac{\delta}{2}-\frac{\beta}{2}+\frac{3}{4} \delta \beta$ : In this case, $\mu^{*}<\mu^{M}<\frac{1}{2}$. From (13), we have

$$
\pi^{M}-\pi^{*}=h(c)=\left(\mu^{M}-\mu^{*}\right)\left(-c\left(\mu^{M}+\mu^{*}\right)+\frac{\delta}{2}\right)+\beta \mu^{M}\left(\delta \mu^{M}-1\right) .
$$

To show $\pi^{M}-\pi^{*}$ is decreasing in $c$, we write $\frac{\partial\left(\pi^{M}-\pi^{*}\right)}{\partial c}$ as

$$
h^{\prime}(c)=\mu^{M^{\prime}}\left(-2 c \mu^{M}+2 \delta \beta \mu^{M}+\frac{\delta}{2}-\beta\right)+\mu^{*^{\prime}}\left(2 c \mu^{*}-\frac{\delta}{2}\right)-\left(\mu^{M^{2}}-\mu^{*^{2}}\right) .
$$

From proposition 1, we know $c \mu^{*}=\frac{\delta}{4}+\frac{\beta}{4}+\frac{\delta \beta}{4} \mu^{*}$. Moreover, the derivation of (22) gives us

$$
\mu^{M^{\prime}}\left(-2 c \mu^{M}+2 \delta \beta \mu^{M}\right)=\mu^{M^{\prime}}\left(\beta \mu^{M}+2 \delta \beta \mu^{M^{2}}\right)+2 \mu^{M^{2}} .
$$

Hence,

$$
h^{\prime}(c)=\mu^{M^{\prime}}\left(2 \delta \beta \mu^{M^{2}}+\beta \mu^{M}+\frac{\delta}{2}-\beta\right)+\mu^{*^{\prime}}\left(\frac{\beta}{2}+\frac{\delta \beta}{2} \mu^{*}\right)+\mu^{M^{2}}+\mu^{*^{2}} .
$$

Since $\mu^{M^{\prime}}=\frac{-2 \mu^{M}}{2 \delta \beta \mu^{M}+\beta+2 c-2 \delta \beta}$, and $\mu^{* \prime}=\frac{-4 \mu^{*}}{4 c-\delta \beta}$, we get

$$
\begin{aligned}
h^{\prime}(c) & =\mu^{M} \frac{-2 \delta \beta \mu^{M^{2}}+\mu^{M}(-\beta+2 c-2 \delta \beta)-\delta+2 \beta}{2 \delta \beta \mu^{M}+\beta+2 c-2 \delta \beta}+\mu^{*} \frac{-2 \beta-2 \delta \beta \mu^{*}+\delta+\beta}{4 c-\delta \beta} \\
& =\mu^{M} \frac{-3 \delta \beta \mu^{M^{2}}-2 \beta \mu^{M}-\frac{\delta}{2}+2 \beta}{2 \delta \beta \mu^{M}+\beta+2 c-2 \delta \beta}+\mu^{*} \frac{-\beta-2 \delta \beta \mu^{*}+\delta}{4 c-\delta \beta},
\end{aligned}
$$

where for the second equality, we use (22). The left term on the R.H.S. of the equality is always negative since

$$
\begin{aligned}
-3 \delta \beta \mu^{M^{2}}-2 \beta \mu^{M}-\frac{\delta}{2}+2 \beta & <-3 \delta \beta\left(\frac{1}{\delta}\right)^{2}-2 \beta \frac{1}{\delta}-\frac{\delta}{2}+2 \beta \\
& =\left(\frac{1}{\delta}\right)\left(-\frac{\delta^{2}}{2}+2 \delta \beta-5 \beta\right)<0
\end{aligned}
$$

where the first and last inequalities are implied by $\delta \mu^{M}>1$ from Lemma 4 . By the way, we often use $\delta>\frac{1}{\mu^{M}} \geq \frac{1}{2}$. Therefore, we assume the right term, $\mu^{*} \frac{-\beta-2 \delta \beta \mu^{*}+\delta}{4 c-\delta \beta}$, is positive; otherwise $h(c)$ 
is decreasing in $c$, and the proof is done. Hence,

$$
h^{\prime}(c)<\mu^{M} \frac{-3 \delta \beta \mu^{M^{2}}-2 \beta \mu^{M}-\frac{\delta}{2}+2 \beta}{2 \delta \beta \mu^{M}+\beta+2 c-2 \delta \beta}+\mu^{M} \frac{-\beta-2 \delta \beta \mu^{*}+\delta}{4 c-\delta \beta},
$$

or equivalently

$$
\frac{h^{\prime}(c)}{\mu^{M}}<\frac{-3 \delta \beta \mu^{M^{2}}-2 \beta \mu^{M}-\frac{\delta}{2}+2 \beta}{2 \delta \beta \mu^{M}+\beta+2 c-2 \delta \beta}+\frac{-\beta-2 \delta \beta \mu^{*}+\delta}{4 c-\delta \beta} .
$$

We also know the left term on the R.H.S. of the inequality is decreasing in $\mu^{M}$ (see the online appendix at the end of the manuscript for the proof). Therefore, we can write

$$
\begin{aligned}
\frac{h^{\prime}(c)}{\mu^{M}} & <\frac{-3 \delta \beta\left(\frac{1}{\delta}\right)^{2}-2 \beta\left(\frac{1}{\delta}\right)-\frac{\delta}{2}+2 \beta}{2 \delta \beta\left(\frac{1}{\delta}\right)+\beta+2 c-2 \delta \beta}+\frac{-\beta-2 \delta \beta \mu^{*}+\delta}{4 c-\delta \beta} \\
& =\left(\frac{1}{\delta}\right) \frac{-\frac{\delta^{2}}{2}+2 \delta \beta-5 \beta}{3 \beta+2 c-2 \delta \beta}+\frac{-\beta-2 \delta \beta \mu^{*}+\delta}{4 c-\delta \beta} .
\end{aligned}
$$

Hence,

$$
\begin{aligned}
\frac{\delta h^{\prime}(c)}{\mu^{M}} & <\frac{-\delta^{2}+4 \delta \beta-10 \beta}{6 \beta+4 c-4 \delta \beta}+\frac{-\delta \beta-2 \delta^{2} \beta \mu^{*}+\delta^{2}}{4 c-\delta \beta} \\
& <\frac{-\delta^{2}+4 \delta \beta-10 \beta}{4 c-\delta \beta}+\frac{-\delta \beta-2 \delta^{2} \beta \mu^{*}+\delta^{2}}{4 c-\delta \beta} \\
& =\left(\frac{1}{4 c-\delta \beta}\right)\left(3 \delta \beta-10 \beta-2 \delta^{2} \beta \mu^{*}\right) .
\end{aligned}
$$

To show $3 \delta \beta-10 \beta-2 \delta^{2} \beta \mu^{*}$ is negative, we first prove $\delta \mu^{*}>\frac{3}{4}$ if $\delta \mu^{M}>1$.

$$
\delta \mu^{M}>1 \Rightarrow Q\left(\frac{1}{\delta}\right)>0 \Rightarrow c<\frac{\delta^{2}}{4}+\delta \beta-\beta=\left(\frac{\delta^{2}}{3}+\frac{7}{12} \delta \beta\right)+\left(-\frac{\delta^{2}}{12}+\frac{5}{12} \delta \beta-\beta\right) .
$$

The last term in the equality is negative for all $\delta$. Therefore,

$$
c<\frac{\delta^{2}}{3}+\frac{7}{12} \delta \beta \Rightarrow \delta \mu^{*}>\frac{3}{4} .
$$

$\delta \mu^{*}>\frac{3}{4}$ implies $3 \delta \beta-10 \beta-2 \delta^{2} \beta \mu^{*}$ is decreasing with $\delta$. As a result $3 \delta \beta-10 \beta-2 \delta^{2} \beta \mu^{*}<$ $3(2) \beta-10 \beta-2(2)^{2} \beta \mu^{*}<0$. This implies $h^{\prime}(c)$ is negative, or equivalently $h(c)$ is decreasing in $c$.

b) $\frac{\delta}{2}+\frac{\beta}{2}+\frac{\delta \beta}{4} \leq c<\frac{\delta}{2}-\frac{\beta}{2}+\frac{3}{4} \delta \beta$ : In this case, $\frac{\delta+\beta}{4 c-\delta \beta}=\mu^{*}<\mu^{M}=\frac{1}{2}$. We can write (13) as: 


$$
h(c)=\frac{1}{4}(\delta \beta-c)+\frac{1}{2}\left(\frac{\delta}{2}-\beta\right)-\frac{\delta}{2} \mu^{*}+c \mu^{*^{2}} .
$$

Hence,

$$
\begin{aligned}
h^{\prime}(c)=-\frac{1}{4}-\frac{\delta}{2} \mu^{*^{\prime}}+2 c \mu^{*} \mu^{*^{\prime}}+\mu^{*^{2}} & =-\frac{1}{4}+\mu^{*^{2}}+\mu^{*^{\prime}}\left(-\frac{\delta}{2}+2 c \mu^{*}\right) \\
& =-\frac{1}{4}+\mu^{*^{2}}+\mu^{*^{\prime}}\left(\frac{\beta}{2}+\frac{\delta \beta}{2} \mu^{*}\right)<0,
\end{aligned}
$$

where the inequality is obtained from $-\frac{1}{4}+\mu^{*^{2}}<0$, and $\mu^{*^{\prime}}<0$. So far we have shown $\pi^{M}-\pi^{*}$ is strictly decreasing with $c$. To prove (ii) it is sufficient to show $\pi^{M}-\pi^{*}$ gets both positive and negative values for some values of $c$. For $c=\frac{\delta}{2}+\frac{\beta}{2}+\frac{\delta \beta}{4}, \mu^{M}=\mu^{*}=\frac{1}{2}$. Thus, $\pi^{M}-\pi^{*}=\frac{\beta}{2}\left(\frac{\delta}{2}-1\right)>0$. We also know, for $c=\frac{\delta^{2}}{4}+\delta \beta-\beta, \mu^{*}<\mu^{M}=\frac{1}{\delta}$, and

$$
\begin{aligned}
\pi^{M}-\pi^{*} & =\left(\mu^{M}-\mu^{*}\right)\left(-c\left(\mu^{M}+\mu^{*}\right)+\frac{\delta}{2}\right) \\
& <\left(\mu^{M}-\mu^{*}\right)\left(-2 c \mu^{*}+\frac{\delta}{2}\right) \\
& =\left(\mu^{M}-\mu^{*}\right)\left(-\frac{\delta \beta}{2} \mu^{*}-\frac{\beta}{2}\right)<0
\end{aligned}
$$

\subsection{Proof of Proposition 8}

Proof. (i) We prove newspaper 1 is always better off to invest on disjoint set of issues (maximum differentiation) for any given $\left(\mu_{1}, \mu_{2}\right)$. Because of the introduction of $u_{T}$, we should modify $(18)$ :

$$
\begin{aligned}
\pi_{1}\left(s_{1}\right) & =\delta \alpha_{1} \mu\left(s_{1}\right)+\alpha_{1}+\delta\left(1-\alpha_{1}-\alpha_{2}\right)\left(\mu\left(s_{1}\right)-\frac{1}{2} \mu\left(s_{1} \cap s_{2}\right)\right)-c \mu\left(s_{1}\right)^{2} \\
& =h\left(\mu\left(s_{1}\right), \mu\left(s_{2}\right)\right)+\frac{\delta \beta \mu\left(s_{1} \cap s_{2}\right)}{1-\left(\mu\left(s_{1}\right)-\mu\left(s_{2}\right)\right)^{2}}\left[\mu\left(s_{1} \cap s_{2}\right)-g\left(\mu\left(s_{1}\right), \mu\left(s_{2}\right)\right)\right],
\end{aligned}
$$

where

$$
h\left(\mu\left(s_{1}\right), \mu\left(s_{2}\right)\right)=\frac{1}{2}+\frac{\delta}{2} \mu\left(s_{1}\right)-\frac{1}{t} \frac{\mu\left(s_{2}\right) \Delta u+u_{T}}{1+\mu\left(s_{2}\right)-\mu\left(s_{1}\right)}+\frac{\delta \mu\left(s_{1}\right)}{t} \frac{\mu\left(s_{1}\right) \Delta u+u_{T}}{1+\mu\left(s_{1}\right)-\mu\left(s_{2}\right)}-c \mu\left(s_{1}\right)^{2}
$$




$$
g\left(\mu\left(s_{1}\right), \mu\left(s_{2}\right)\right)=-\frac{3}{2} \mu\left(s_{1}\right)^{2}+\mu\left(s_{1}\right)\left(2 \mu\left(s_{2}\right)-\frac{1}{\delta}+\frac{3}{2}\right)+\left(1-\mu\left(s_{2}\right)\right)\left(\frac{1}{2} \mu\left(s_{2}\right)-\frac{1}{\delta}\right)+\frac{u_{T}}{\Delta u} .
$$

There are two cases:

a) $\mu_{1} \leq \mu_{2}$ : Max differentiation is a dominant strategy if and only if $\mu_{1} \leq g\left(\mu_{1}, \mu_{2}\right)$, or equivalently:

$$
a\left(\mu_{1}, \mu_{2}\right)=-\frac{3}{2} \mu_{1}^{2}+\mu_{1}\left(2 \mu_{2}-\frac{1}{\delta}+\frac{1}{2}\right)+\left(1-\mu_{2}\right)\left(\frac{1}{2} \mu_{2}-\frac{1}{\delta}\right)+\frac{u_{T}}{\Delta u} \geq 0 .
$$

The right term, $a\left(0, \mu_{2}\right)=\left(1-\mu_{2}\right)\left(\frac{1}{2} \mu_{2}-\frac{1}{\delta}\right)+\frac{u_{T}}{\Delta u}$, is positive, as long as $\frac{u_{T}}{\Delta u} \geq \frac{1}{\delta}$. And $a\left(\mu_{2}, \mu_{2}\right)>0$, if $\mu_{2}-\frac{1}{\delta}+\frac{u_{T}}{\Delta u}>0$. Therefore, if $\frac{u_{T}}{\Delta u} \geq \frac{1}{\delta}$ maximum differentiation is a dominant strategy for any given $\left(\mu_{1}, \mu_{2}\right)$ satisfying $\mu_{1} \leq \mu_{2}$.

b) $\mu_{1} \geq \mu_{2}$ : Newspaper 1 prefers maximum differentiation if and only if $\mu_{2} \leq g\left(\mu_{1}, \mu_{2}\right)$. This is equivalent to:

$$
b\left(\mu_{1}, \mu_{2}\right)=-\frac{3}{2} \mu_{1}^{2}+\mu_{1}\left(2 \mu_{2}-\frac{1}{\delta}+\frac{3}{2}\right)+\left(1-\mu_{2}\right)\left(\frac{1}{2} \mu_{2}-\frac{1}{\delta}\right)+\frac{u_{T}}{\Delta u}-\mu_{2} \geq 0 .
$$

$b\left(\mu_{2}, \mu_{2}\right)>0$, as long as $\mu_{2}-\frac{1}{\delta}+\frac{u_{T}}{\Delta u}>0$. Thus, $b\left(\mu_{1}, \mu_{2}\right)>0$ for any given $\left(\mu_{1}, \mu_{2}\right)$ satisfying $\mu_{1} \geq \mu_{2}$, if $b\left(\frac{1}{2}, \mu_{2}\right)>0$.

$$
b\left(\frac{1}{2}, \mu_{2}\right)=-\frac{1}{2} \mu_{2}^{2}+\mu_{2}\left(\frac{1}{2}+\frac{1}{\delta}\right)+\frac{3}{8}-\frac{3}{2 \delta}+\frac{u_{T}}{\Delta u}
$$

$\frac{u_{T}}{\Delta u} \geq \frac{3}{2 \delta}$ implies $b\left(\frac{1}{2}, \mu_{2}\right)>0$.

To conclude, $\frac{u_{T}}{\Delta u} \geq \frac{3}{2 \delta}$ is the sufficient condition for the dominance of maximum differentiation for any given $\left(\mu_{1}, \mu_{2}\right)$.

(ii) In this part, we will show $\frac{\partial^{2} \pi_{1}}{\partial \mu_{1} \partial \mu_{2}}>0$.

$$
\frac{\partial^{2} \pi_{1}}{\partial \mu_{1} \partial \mu_{2}}=\frac{1}{t}\left[\frac{-\left(1-\mu_{1}-\mu_{2}\right) \Delta u+2 u_{T}}{\left(1-\mu_{1}+\mu_{2}\right)^{2}}+\delta \frac{u_{T}\left(1-\mu_{2}-\mu_{1}\right)+2 \Delta u \mu_{1}\left(1-\mu_{1}\right)}{\left(1+\mu_{1}-\mu_{2}\right)^{3}}\right]
$$

The right term is positive. Since $2 u_{T}>\Delta u$, the left term is also positive.

(iii) First, we show the best response of newspaper 1 to newspaper 2's quality, $\mu_{2}$, is unique. Second, we compute the symmetric equilibrium. Finally we show the equilibrium quality, $\mu^{T}$, is increasing in $\delta$. 
1) From (25), we can compute the derivatives of newspaper 1's profit.

$$
\begin{gathered}
\pi_{1}^{\prime}\left(s_{1}\right)=\frac{\delta}{2}-\frac{1}{t} \frac{\mu_{2} \Delta u+u_{T}}{\left(1+\mu_{2}-\mu_{1}\right)^{2}}+2 \delta \beta \frac{\mu_{1}}{1+\mu_{1}-\mu_{2}}-\frac{\delta}{t} \frac{\mu_{1}^{2} \Delta u-u_{T}\left(1-\mu_{j}\right)}{\left(1+\mu_{1}-\mu_{2}\right)^{2}}-2 c \mu_{1} \\
\pi_{1}^{\prime \prime}\left(s_{1}\right)=-\frac{2}{t} \frac{\mu_{2} \Delta u+u_{T}}{\left(1+\mu_{2}-\mu_{1}\right)^{3}}+\frac{2 \delta\left(1-\mu_{2}\right)}{t} \frac{\left(1-\mu_{1}-\mu_{2}\right) \Delta u-u_{T}}{\left(1+\mu_{1}-\mu_{2}\right)^{3}}-2 c \\
\pi_{1}^{\prime \prime \prime}\left(s_{1}\right)=-\frac{6}{t} \frac{\mu_{2} \Delta u+u_{T}}{\left(1+\mu_{2}-\mu_{1}\right)^{4}}-\frac{6 \delta\left(1-\mu_{2}\right)}{t} \frac{\left(1-\mu_{1}-\mu_{2}\right) \Delta u-u_{T}}{\left(1+\mu_{1}-\mu_{2}\right)^{4}}
\end{gathered}
$$

There are two cases:

1.1) $u_{T} \geq \Delta u$ : In this case, the profit function is concave regardless of $\mu_{j}$.

1.2) $u_{T}<\Delta u$ : The profit function might be convex for some $\mu_{j}$. Since the third derivative is negative, $\pi^{\prime}\left(s_{1} \mid \mu_{1}=0\right)>0$ would be sufficient to prove that there exists a unique best response.

$$
\begin{aligned}
\pi^{\prime}\left(s_{1} \mid \mu_{1}=0\right) & =\frac{\delta}{2}-\frac{1}{t} \frac{\mu_{2} \Delta u+u_{T}}{\left(1+\mu_{2}\right)^{2}}+\frac{\delta}{t} \frac{u_{T}}{1-\mu_{2}} \\
& >-\frac{1}{t} \frac{\mu_{2} \Delta u+u_{T}}{\left(1+\mu_{2}\right)^{2}}+\frac{1}{t} \frac{u_{T}}{1-\mu_{2}} \\
& =\frac{1}{t\left(1+\mu_{2}\right)^{2}\left(1-\mu_{2}\right)}\left[u_{T}\left(1+\mu_{2}\right)^{2}-u_{T}\left(1-\mu_{2}\right)-\mu_{2} \Delta u\left(1-\mu_{2}\right)\right] \\
& =\frac{1}{t\left(1+\mu_{2}\right)^{2}\left(1-\mu_{2}\right)}\left[u_{T}\left(\mu_{2}+\mu_{2}^{2}\right)+\mu_{2}\left(2 u_{T}-\Delta u\left(1-\mu_{2}\right)\right)\right] \\
& >0 .
\end{aligned}
$$

2) Depending on the value of $\delta$, the best response could take three values, $0, \frac{1}{2}$ or the solution of $\pi^{\prime}\left(\mu_{1}, \mu_{2}\right)=0$. Therefore, the symmetric equilibrium candidates are:

2.1) $(0,0)$ : This is as an equilibrium, if $\pi_{i}^{\prime}\left(s_{i} \mid \mu_{i}=\mu_{j}=0\right)<0$ for $i, j \in\{1,2\}$. This is equivalent to $\delta<\underline{\delta}^{T}=\frac{u_{T} / t}{u_{T} / t+1 / 2}$.

2.2) $(\hat{\mu}, \hat{\mu}) \in\left(0, \frac{1}{2}\right)^{2}$ : For all $\delta$ satisfying $\underline{\delta}^{T} \leq \delta \leq \bar{\delta}^{T}$, we have $\pi_{i}^{\prime}\left(s_{i} \mid \mu_{i}=\mu_{j}=\frac{1}{2}\right)<0<\pi_{i}^{\prime}\left(s_{i} \mid\right.$ $\left.\mu_{i}=\mu_{j}=0\right)$. Therefore, $(\hat{\mu}, \hat{\mu})$ is an equilibrium, where $\hat{\mu}$ is the positive solution of $Q$ :

$$
Q(\mu)=\pi^{\prime}\left(s_{1} \mid \mu_{1}=\mu_{2}=\mu\right)=\mu^{2}(-\delta \beta)+\mu\left(-\beta+2 \delta \beta-2 c-\frac{u_{T} \delta}{t}\right)+\frac{\delta}{2}+\frac{u_{T}}{t}(\delta-1) .
$$

2.3) $\left(\frac{1}{2}, \frac{1}{2}\right)$ : This is an equilibrium, if $\pi_{i}^{\prime}\left(s_{i} \mid \mu_{i}=\mu_{j}=\frac{1}{2}\right)>0$ for $i, j \in\{1,2\}$. This is equivalent to $\delta>\bar{\delta}^{T}=\frac{c+\Delta u / 2 t+u_{T} / t}{u_{T} / 2 t+1 / 2+3 \Delta u / 4 t}$.

3) Now, we prove that $\mu^{T}$ is increasing in $\delta$. For $\delta<\underline{\delta}^{T}, \mu^{T}$ is zero, and for $\delta<\bar{\delta}^{T}, \mu^{T}$ is $1 / 2$. So it is sufficient to prove that $\mu^{T}$ is increasing in $\delta$ for $\delta \in\left[\underline{\delta}^{T}, \bar{\delta}^{T}\right]$. If $\delta \in\left[\underline{\delta}^{T}, \bar{\delta}^{T}\right], \mu^{T}$ is the 
positive solution of

$$
\mu^{T^{2}}(-\delta \beta)+\mu^{T}\left(-\beta+2 \delta \beta-2 c-\frac{u_{T} \delta}{t}\right)+\frac{\delta}{2}+\frac{u_{T}}{t}(\delta-1)=0 .
$$

Hence,

$$
\mu^{T^{\prime}}\left[-2 \mu^{T} \delta \beta-\beta+2 \delta \beta-2 c-u_{T} \delta / t\right]-\beta \mu^{T^{2}}+\mu^{T}\left(2 \beta-u_{T} / t\right)+1 / 2+u_{T} / t=0
$$

As $-2 \mu^{T} \delta \beta-\beta+2 \delta \beta-2 c-u_{T} \delta / t<0$, and $-\beta \mu^{T^{2}}+\mu^{T}\left(2 \beta-u_{T} / t\right)+1 / 2+u_{T} / t>0, \mu^{T^{\prime}}$ is positive.

\subsection{Proof of Proposition 9}

Proof. (i) From the proof of Proposition 6, we can write

$$
\begin{aligned}
2\left(\mu^{T}-\mu^{*}\right)\left(c-\frac{\delta \beta}{4}\right) & =\mu^{T^{2}}(-\delta \beta)+\left(\frac{3 \delta \beta}{2}-\beta-\frac{u_{T} \delta}{t}\right) \mu^{T}-\frac{\beta}{2}+\frac{u_{T}}{t}(\delta-1) \\
& =\delta \beta \mu^{T}\left(\frac{1}{2}-\mu^{T}\right)+\beta \mu^{T}\left(\frac{\delta}{2}-1\right)+\frac{\beta}{2}\left(\mu^{T} \delta-1\right)+\frac{u_{T}}{t}\left(\delta-1-\delta \mu_{T}\right) \\
& \geq 0,
\end{aligned}
$$

where we have $\delta>\frac{1}{\mu^{T}}>2$, to prove the inequality.

(ii) Please see the online appendix at the end of the manuscript.

(iii) The proof is the same as Proposition 7 (iii).

\subsection{Proofs of Propositions 10 and 11}

Please see the online appendix at the end of the manuscript. 


\section{Appendix B}

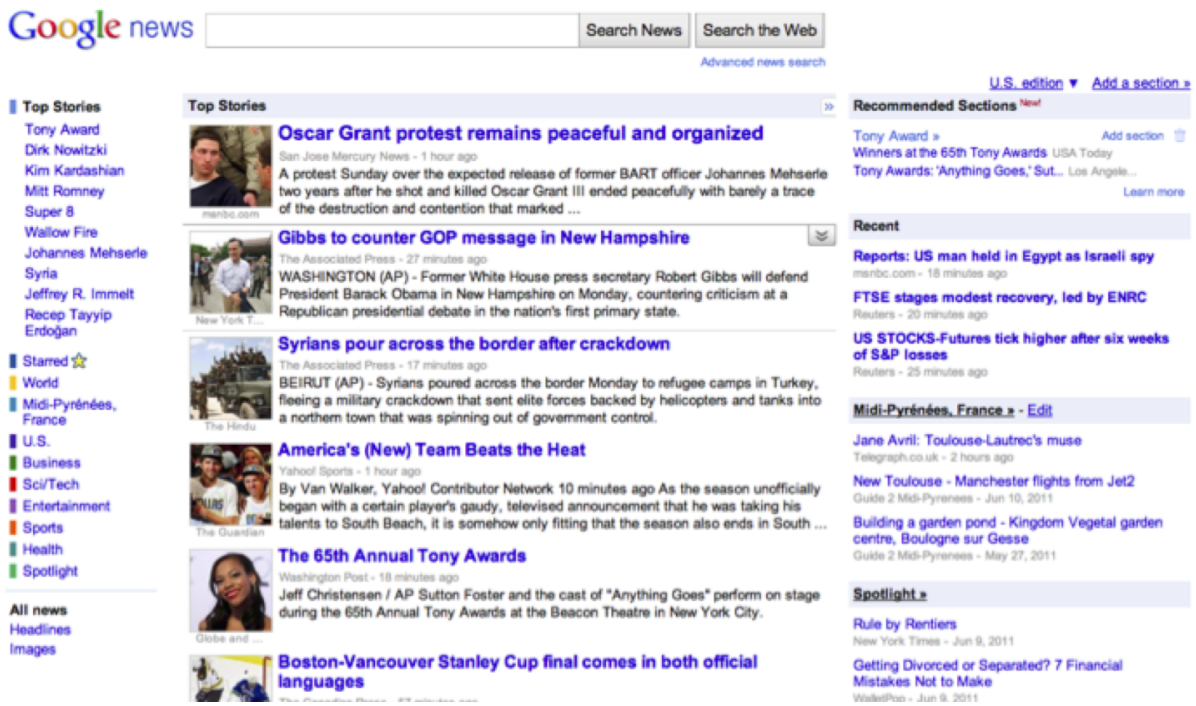

Figure 7: The Google News 


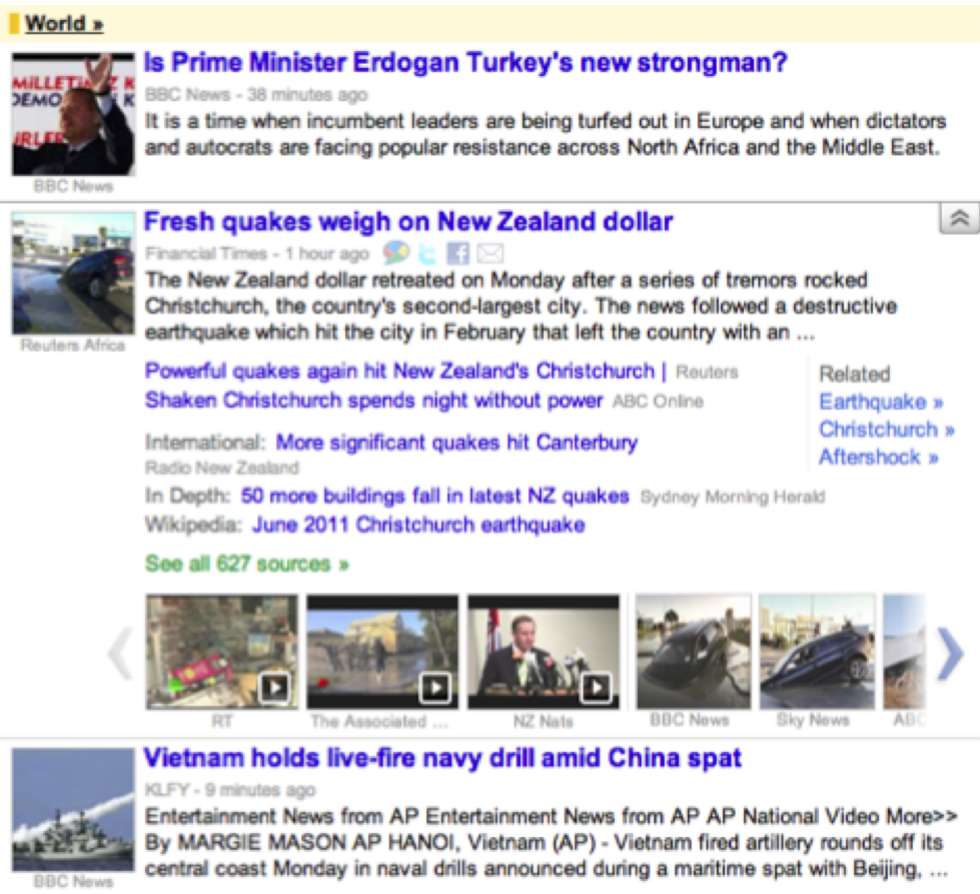

Figure 8: An article from Financial Times in the Google News. There is a short abstract of the article in the two or three lines, and a link to the original article. 


\section{Supplementary materials: not for publication}

\subsection{Proof of Proposition 7 (ii)}

Claim 1. $f\left(\mu^{M}\right)=\frac{-3 \delta \beta \mu^{M^{2}}-2 \beta \mu^{M}-\frac{\delta}{2}+2 \beta}{2 \delta \beta \mu^{M}+\beta+2 c-2 \delta \beta}$ is decreasing in $\mu^{M}$.

Proof.

$$
\begin{aligned}
f^{\prime}\left(\mu^{M}\right) & =\frac{-6 \delta^{2} \beta^{2} \mu^{M^{2}}-6 \delta \beta^{2} \mu^{M}-12 \delta \beta c \mu^{M}+12 \delta^{2} \beta^{2} \mu^{M}-2 \beta^{2}-4 \beta c+\delta^{2} \beta}{\left(2 \delta \beta \mu^{M}+\beta+2 c-2 \delta \beta\right)^{2}} \\
& =\frac{-2 \delta^{2} \beta-2 \beta^{2}-4 c \beta}{\left(2 \delta \beta \mu^{M}+\beta+2 c-2 \delta \beta\right)^{2}}<0,
\end{aligned}
$$

where the last equality is implied by $(22), 2 c \mu^{M}=\mu^{M^{2}}(-\delta \beta)+\mu^{M}(-\beta+2 \delta \beta)+\frac{\delta}{2}$.

\subsection{Extension to imperfect certification technology}

The reader's utility from aggregator is:

$$
\begin{aligned}
U^{A g g}(x) & =\mu\left(s_{1}-s_{2}\right)\left(\frac{1+\Delta P}{2}\left(\Delta u+u_{0}-x t\right)+\frac{1-\Delta P}{2}\left(u_{0}-(1-x) t\right)\right) \\
& +\mu\left(s_{2}-s_{1}\right)\left(\frac{1+\Delta P}{2}\left(\Delta u+u_{0}-(1-x) t\right)+\frac{1-\Delta P}{2}\left(u_{0}-x t\right)\right) \\
& +\mu\left(s_{2} \cap s_{1}\right)\left(\Delta u+u_{0}-\frac{t}{2}\right)+\left(1-\mu\left(s_{2} \cup s_{1}\right)\right)\left(u_{0}-\frac{t}{2}\right) \\
& =u_{0}+\Delta u\left(\frac{1+\Delta P}{2} \mu\left(s_{2} \cup s_{1}\right)+\frac{1-\Delta P}{2} \mu\left(s_{2} \cap s_{1}\right)\right) \\
& +\Delta P\left(\mu_{2}-\mu_{1}\right) x t-\frac{t}{2}\left(1+\Delta P\left(\mu_{2}-\mu_{1}\right)\right) \\
& =u_{0}+\frac{\Delta u}{2}\left(\mu_{1}+\mu_{2}+\Delta P\left(\mu_{1}+\mu_{2}-2 \mu_{12}\right)\right) \\
& +\Delta P\left(\mu_{2}-\mu_{1}\right) x t-\frac{t}{2}\left(1+\Delta P\left(\mu_{2}-\mu_{1}\right)\right)
\end{aligned}
$$

The utility form newspaper 1 is not affected by $\Delta P$.

$$
U^{1}(x)=u_{0}+\mu\left(s_{1}\right) \Delta u-x t
$$

Therefore, market share of newspaper 1 is given by:

$$
0<\alpha_{1}=\frac{1}{2}-\frac{\beta}{2} \frac{\mu_{2}-\mu_{1}+\Delta P\left(\mu_{1}+\mu_{2}-2 \mu_{12}\right)}{1+\Delta P\left(\mu_{2}-\mu_{1}\right)} \leq \frac{1}{2},
$$


and by computing $\partial \alpha_{1} / \partial \mu_{1}$, we can show there exists a unique threshold $\hat{P}$ in $(0,1)$ such that $\partial \alpha_{1} / \partial \mu_{1} \leq 0$ if and only if $\Delta P \geq \hat{P}$.

$$
\frac{\partial \alpha_{1}}{\partial \mu_{1}}=\frac{\beta}{2} \frac{-2(\Delta P)^{2}\left(\mu_{2}-\mu_{12}\right)-\Delta P+1}{\left(1+\Delta P\left(\mu_{2}-\mu_{1}\right)\right)^{2}} .
$$

The profit of newspaper 1 is:

$$
\begin{aligned}
\pi_{1} & =\left(1+\delta \mu_{1}\right) \alpha_{1}+\delta\left(1-\alpha_{1}-\alpha_{2}\right)\left(P_{H} \mu\left(s_{1}-s_{2}\right)+\frac{1}{2} \mu\left(s_{2} \cap s_{1}\right)\right)-c \mu_{1}^{2} \\
& =\left(1+\delta \mu_{1}\right) \alpha_{1}+\frac{\delta}{2}\left(1-\alpha_{1}-\alpha_{2}\right)\left(\mu_{1}+\Delta P\left(\mu_{1}-\mu_{12}\right)\right)-c \mu_{1}^{2} \\
& =h\left(\mu_{1}, \mu_{2}\right)+\mu_{12} g\left(\mu_{1}, \mu_{2}, \mu_{12}\right)
\end{aligned}
$$

where

$$
\begin{aligned}
h\left(\mu_{1}, \mu_{2}\right) & =\frac{1}{2}+\frac{\delta}{2} \mu_{1}-\frac{\beta}{2} \frac{\mu_{2}-\mu_{1}+\Delta P\left(\mu_{1}+\mu_{2}\right)}{1+\Delta P\left(\mu_{2}-\mu_{1}\right)}-\frac{\delta \beta}{4} \frac{\mu_{2}-\mu_{1}+\Delta P\left(\mu_{1}+\mu_{2}\right)}{1+\Delta P\left(\mu_{2}-\mu_{1}\right)}(1-\Delta P) \mu_{1} \\
& +\frac{\delta \beta}{4} \frac{\mu_{1}-\mu_{2}+\Delta P\left(\mu_{1}+\mu_{2}\right)}{1+\Delta P\left(\mu_{1}-\mu_{2}\right)}(1+\Delta P) \mu_{1}-c \mu_{1}^{2},
\end{aligned}
$$

and

$$
\begin{aligned}
g\left(\mu_{1}, \mu_{2}, \mu_{12}\right) & =\frac{\beta \Delta P}{1+\Delta P\left(\mu_{2}-\mu_{1}\right)}\left(1+\delta \mu_{1}\right) \\
& -\frac{\delta \beta \Delta P}{4}\left(\frac{\mu_{2}-\mu_{1}+\Delta P\left(\mu_{1}+\mu_{2}\right)}{1+\Delta P\left(\mu_{2}-\mu_{1}\right)}+\frac{\mu_{1}-\mu_{2}+\Delta P\left(\mu_{1}+\mu_{2}\right)}{1+\Delta P\left(\mu_{1}-\mu_{2}\right)}\right) \\
& -\frac{\delta \beta \Delta P}{2}\left(\frac{1}{1+\Delta P\left(\mu_{2}-\mu_{1}\right)}+\frac{1}{1+\Delta P\left(\mu_{1}-\mu_{2}\right)}\right)\left(\mu_{1}+\Delta P\left(\mu_{1}-\mu_{12}\right)\right) \\
& =\frac{\delta \beta \Delta P^{2}}{2}\left(\frac{1}{1+\Delta P\left(\mu_{2}-\mu_{1}\right)}+\frac{1}{1+\Delta P\left(\mu_{1}-\mu_{2}\right)}\right) \mu_{12}+\text { cst. }
\end{aligned}
$$

Therefore, $\frac{\partial^{2} \pi_{1}}{\partial \mu_{12}^{2}}>0$. This extends Proposition 2 .

To extend Lemma 4, we first note $g(\mu, \mu, \mu)=\beta \Delta P(1-\Delta P \delta \mu)$. Therefore, if $(\mu, \mu)$ is the maximum differentiation equilibrium, then

$$
\pi_{1 \mid \max }=h(\mu, \mu)>\pi_{1 \mid \min }=h(\mu, \mu)+\mu g(\mu, \mu, \mu) \Longrightarrow g(\mu, \mu, \mu)<0 \Longrightarrow \Delta P \delta \mu>1 .
$$

Similarly, if $(\mu, \mu)$ is the minimum differentiation equilibrium, then

$$
\pi_{1 \mid \min }=h(\mu, \mu)+\mu g(\mu, \mu, \mu)>\pi_{1 \mid \max }=h(\mu, \mu) \Longrightarrow g(\mu, \mu, \mu)>0 \Longrightarrow \Delta P \delta \mu<1 .
$$


Proposition 4 extends as follows. The equilibrium quality in the maximum differentiation equilibrium is $\mu^{M}=\frac{1}{2}$ for $c<\frac{-\delta \beta \Delta P^{2}}{4}+\frac{1}{2}\left(-\beta \Delta P^{2}+\frac{\delta \beta}{2}\left(1+3 \Delta P^{2}\right)\right)+\frac{\delta}{2}+\frac{\beta}{2}(1-\Delta P)$. Otherwise, $\mu^{M}$ is the positive solution of

$$
Q(\mu)=\left(-\delta \beta \Delta P^{2}\right) \mu^{2}+\left(-\beta \Delta P^{2}+\frac{\delta \beta}{2}\left(1+3 \Delta P^{2}\right)-2 c\right) \mu+\frac{\delta}{2}+\frac{\beta}{2}(1-\Delta P)=0 .
$$

In the maximum differentiation equilibrium, the profit of each newspaper is given by

$$
\frac{1}{2}+\frac{\delta}{2} \mu^{M}-\beta \Delta P \mu^{M}+\delta \beta\left(\Delta P \mu^{M}\right)^{2}-c\left(\mu^{M}\right)^{2}
$$

On the other hand, we know $2 c \mu^{*}-\frac{\beta}{2}-\frac{\delta \beta}{2} \mu^{*}-\frac{\delta}{2}=0$. By adding this to (33), we get

$$
2\left(\mu^{M}-\mu^{*}\right)\left(c-\frac{\delta \beta}{4}\right)=\frac{\beta \Delta P}{2}\left(\Delta P \delta \mu^{M}-1\right)+\Delta P^{2} \beta \mu^{M}\left(\delta-1-\delta \mu^{M}\right) \geq 0,
$$

where we use $\Delta P \delta \mu^{M}>1$ to prove that the first term is positive. For the second term to be positive it is sufficient to have $\delta \geq 2$ since $\delta-1-\delta \mu^{M} \geq \frac{\delta}{2}-1$. And $\delta \geq 2$ is implied by $\delta>\frac{1}{\Delta P \mu^{M}} \geq 2$. As a result, the aggregator improves the quality in the case of maximum differentiation, $\mu^{M}>\mu^{*}$.

We now show how quality is affected by $\Delta P$ in the maximum differentiation equilibrium. We have:

$$
\begin{aligned}
\frac{\partial Q}{\partial \Delta P} & =\frac{\partial \mu^{M}}{\partial \Delta P}\left(-\frac{1}{2} \delta \beta \Delta P^{2}-\beta \Delta P^{2}+\frac{\delta \beta}{2}-2 c\right) \\
& +2 \beta \Delta P\left(-\delta \mu^{M}-1+\frac{5}{4} \delta\right) \mu^{M}+\frac{\beta}{2}\left(\Delta P \delta \mu^{M}-1\right)=0
\end{aligned}
$$

The second term in the first line is negative since $c>\frac{\delta \beta}{4}$. The term in the second line is positive since $\Delta P \delta \mu^{M}>1$, and $-\delta \mu^{M}-1+\frac{5}{4} \delta>\frac{3 \delta}{4}-1>0$. This implies $\frac{\partial \mu^{M}}{\partial \Delta P}>0$.

We find that the effect of $\Delta P$ on newspapers' profits in the maximum differentiation equilibrium is ambiguous. Using the envelope theorem, we find:

$$
\frac{d \pi_{1}}{d \Delta P}=\frac{\partial \pi_{1}}{\partial \mu_{1}^{M}} \frac{\partial \mu_{1}^{M}}{\partial \Delta P}+\frac{\partial \pi_{1}}{\partial \mu_{2}^{M}} \frac{\partial \mu_{2}^{M}}{\partial \Delta P}+\frac{\partial \pi_{1}}{\partial \Delta P}=\frac{\partial \pi_{1}}{\partial \mu_{2}^{M}} \frac{\partial \mu_{2}^{M}}{\partial \Delta P}+\frac{\partial \pi_{1}}{\partial \Delta P}
$$

The direct effect for given quality of newspapers is positive (i.e., $\frac{\partial \pi_{1}}{\partial \Delta P}>0$ ) since newspapers benefit more from readership-expansion effect. However, the indirect effect through the rival's 
quality increase has an ambiguous sign due to $\partial \pi_{1} / \partial \mu_{2}^{M}$. We can write

$$
\frac{\partial \pi_{1}}{\partial \mu_{2}^{M}}=\left(1+\delta \mu_{1}\right) \frac{\partial \alpha_{1}}{\partial \mu_{2}^{M}}+\frac{\delta}{2} \mu_{1}(1+\Delta P) \frac{\partial \alpha_{A g g}}{\partial \mu_{2}^{M}}
$$

The aggregator's market share increases with the quality of newspaper 2 (i.e., $\frac{\partial \alpha_{A g g}}{\partial \mu_{2}^{M}}>0$ ) while 1's market share decreases with the rival's quality (i.e., $\frac{\partial \alpha_{1}}{\partial \mu_{2}^{M}}<0$ ). As $\Delta P$ increases, the former is more likely to dominate the latter such that for large $\Delta P, \frac{\partial \pi_{1}}{\partial \mu_{2}^{M}}$ is positive.

\subsection{Proof of Proposition 9 (ii)}

Proof. If newspaper $i$ opts out its best deviation quality would be $\mu_{i}=\frac{\delta+\beta-\frac{\delta\left(\mu^{T} \Delta u+u_{T}\right)}{t}}{4 c-2 \delta \beta}$. And its market share changes from $\alpha_{T}=\frac{1}{2}-\beta \mu^{T}-\frac{u_{T}}{t}$ to $\alpha_{i}=\frac{1}{2}-\frac{\left(\mu^{T}-\mu_{i}\right) \Delta u+u_{T}}{2 t}$. As a result, the gain from deviation is

$$
\begin{array}{r}
d\left(\mu_{i}, \mu^{T}\right)=\alpha_{i}\left(1+\delta \mu_{i}\right)-c \mu_{i}^{2}-\alpha_{T}\left(1+\delta \mu^{T}\right)-2 \delta\left(\frac{u_{T}}{t}+\beta \mu^{T}\right) \mu^{T}+c \mu^{T^{2}} \\
\left(\alpha_{i}-\alpha_{T}\right)\left(1+\delta \mu_{i}\right)-\left(\mu^{T}-\mu_{i}\right)\left[-c\left(\mu^{T}+\mu_{i}\right)+\delta \alpha_{T}\right]-2 \frac{\delta}{t}\left(u_{T}+\mu^{T} \Delta u\right) \mu^{T}= \\
\left(\alpha_{i}-\alpha_{T}\right)+\frac{\delta u_{T}}{2 t} \mu_{i}+\frac{\delta \beta}{2} \mu_{i} \mu^{T}+\frac{\delta \beta}{2} \mu_{i}^{2}-\left(\mu^{T}-\mu_{i}\right)\left[-c\left(\mu^{T}+\mu_{i}\right)+\delta \alpha_{T}\right]-2 \frac{\delta}{t}\left(u_{T}+\mu^{T} \Delta u\right) \mu^{T}= \\
\left(\alpha_{i}-\alpha_{T}\right)+\frac{\delta \beta}{2} \mu_{i} \mu^{T}-\left(\mu^{T}-\mu_{i}\right)\left[-c\left(\mu^{T}+\mu_{i}\right)+\delta \alpha_{T}+\frac{\delta u_{T}}{2 t}+\frac{\delta \beta}{2}\left(\mu_{i}+\mu^{T}\right)\right]-\frac{3 \delta}{2 t}\left(u_{T}+\mu^{T} \Delta u\right) \mu^{T}= \\
\frac{1}{2 t}\left(\mu^{T} \Delta u+u_{T}+\mu_{i} \Delta u\right)+\frac{1}{2 t}\left(\delta \mu_{i} \mu^{T} \Delta u\right)-\frac{3}{2 t} \delta \mu^{T}\left(\mu^{T} \Delta u+u_{T}\right)- \\
\left(\mu^{T}-\mu_{i}\right)\left[-c\left(\mu_{i}+\mu^{T}\right)+\delta / 2-\frac{\delta\left(\mu^{T}-\mu_{i}\right) \Delta u}{2 t}-\frac{\delta u_{T}}{2 t}\right] .
\end{array}
$$

By adding $-\frac{1}{2} Q\left(\mu^{T}\right)$ (from (32)), and $c \mu_{i}=\delta+\beta-\frac{\delta\left(\mu^{T} \Delta u+u_{T}\right)}{t}+\frac{\delta \beta}{2} \mu_{i}$ to the last term, we get:

$$
\begin{array}{r}
(2 t) d\left(\mu_{i}, \mu^{T}\right)=\left(\mu^{T} \Delta u+u_{T}+\mu_{i} \Delta u\right)+\delta \mu_{i} \mu^{T} \Delta u-3 \delta \mu^{T}\left(\mu^{T} \Delta u+u_{T}\right) \\
-\left(\mu^{T}-\mu_{i}\right)\left[\mu^{T^{2}} \delta \Delta u+\mu^{T}\left(\Delta u+\delta u_{T}\right)-\frac{5}{2} \mu^{T} \delta \Delta u-\frac{3}{2} u_{T} \delta+u_{T}-\Delta u / 2\right] .
\end{array}
$$

We can rearrange it to

$$
\begin{array}{r}
(2 t) d\left(\mu_{i}, \mu^{T}\right)=u_{T}+2 \mu^{T} \Delta u-2 \delta \mu^{T^{2}} \Delta u-3 \delta \mu^{T} u_{T} \\
-\left(\mu^{T}-\mu_{i}\right)\left[\mu^{T^{2}} \delta \Delta u+\mu^{T}\left(\Delta u+\delta u_{T}-\frac{3}{2} \delta \Delta u\right)-\frac{3}{2} u_{T} \delta+u_{T}+\Delta u / 2\right] .
\end{array}
$$


From (32), we know $-\mu^{T}(4 c-2 \delta \beta)=\mu^{T^{2}}(2 \delta \beta)+2 \mu^{T}\left(\beta-\delta \beta+\frac{u_{T} \delta}{t}\right)-\delta-\frac{2 u_{T}}{t}(\delta-1)$. Also $\mu_{i}(4 c-2 \delta \beta)=\delta+\beta-\frac{\delta\left(\mu^{T} \Delta u+u_{T}\right)}{t}$. Adding them up gives us

$$
\left(\mu_{i}-\mu^{T}\right)(4 c-2 \delta \beta)=\frac{1}{t}\left[2 \mu^{T^{2}} \delta \Delta u+2 \mu^{T}\left(\Delta u-\frac{3}{2} \delta \Delta u+u_{T} \delta\right)-3 u_{T} \delta+2 u_{T}+\Delta u\right] .
$$

Hence, the gain ${ }^{25}$ is

$$
u_{T}+2 \mu^{T} \Delta u-2 \delta \mu^{T^{2}} \Delta u-3 \delta \mu^{T} u_{T}+\frac{t}{2}(4 c-2 \delta \beta)\left(\mu^{T}-\mu_{i}\right)^{2},
$$

or equivalently

$$
\mu^{T^{2}}\left(\frac{t}{2}(4 c-2 \delta \beta)-2 \delta \Delta u\right)+\mu^{T}\left(2 \Delta u-3 \delta u_{T}-t \mu_{i}(4 c-2 \delta \beta)\right)+u_{T}+\frac{t}{2}(4 c-2 \delta \beta) \mu_{i}^{2}
$$

We first show the gain is decreasing in $\mu^{T}$, and then it is negative for $\mu^{T}=\mu^{*}$. Therefore, opt-out is not profitable for $\mu^{T} \geq \mu^{*}$.

Claim 2. The gain from opt-out is decreasing in $\mu^{T}$ :

The derivative of the gain with respect to $\mu^{T}$ is

$$
t(4 c-2 \delta \beta)\left(\mu^{T}-\mu_{i}\right)-4 \delta \Delta u \mu^{T}+2 \Delta u-3 \delta u_{T}-t \mu^{T}(4 c-2 \delta \beta) \mu_{i}^{\prime}+t \mu_{i}(4 c-2 \delta \beta) \mu_{i}^{\prime} .
$$

We can replace $(4 c-2 \delta \beta) \mu_{i}^{\prime}$ by $-\delta \beta$ and $t(4 c-2 \delta \beta)\left(\mu^{T}-\mu_{i}\right)$ from (35). Hence,

$-2 \mu^{T^{2}} \delta \Delta u-2 \mu^{T} \Delta u+3 \delta \Delta u \mu^{T}-2 \delta u_{T} \mu^{T}+3 u_{T} \delta-2 u_{T}-\Delta u-3 \delta \Delta u \mu^{T}+2 \Delta u-3 \delta u_{T}-\delta \Delta u \mu_{i}$,

or equivalently

$$
-2 \mu^{T^{2}} \delta \Delta u-2 \mu^{T} \Delta u-2 \delta u_{T} \mu^{T}-2 u_{T}+\Delta u-\delta \Delta u \mu_{i}
$$

which is negative since $2 u_{T} \geq \Delta u$.

Claim 3. The gain from opt-out is negative for $\mu^{T}=\mu^{*}$ :

\footnotetext{
${ }^{25}$ Since $t$ is a constant, we can consider the gain as $\frac{d\left(\mu_{i}, \mu^{t}\right)}{2 t}$.
} 
We know:

$$
\begin{aligned}
\delta \mu^{T}>1 \Rightarrow Q\left(\frac{1}{\delta}\right)>0 & \Rightarrow c<\frac{\delta^{2}}{4}+\frac{\delta^{2} u_{T}}{2 t}+\frac{\delta}{t}\left(\Delta u-u_{T}\right)-\beta \\
& \Rightarrow c<\frac{\delta^{2}}{4}+\delta \beta-\beta+\frac{\delta^{2} u_{T}}{2 t}-\frac{\delta u_{T}}{t} \\
& \left.\Rightarrow c<\left(\frac{3 \delta^{2}}{8}+\frac{5 \delta \beta}{8}\right)+\left(\delta^{2}\left(-\frac{1}{8}+\frac{u_{T}}{2 t}\right)+\frac{\delta}{t}\left(\frac{3 \Delta u}{8}-u_{T}\right)\right)-\beta\right)
\end{aligned}
$$

The last term is negative, according to A4, and A1'. Therefore,

$$
c<\frac{3 \delta^{2}}{8}+\frac{5 \delta \beta}{8} \Rightarrow \delta \mu^{*}>\frac{2}{3}
$$

If $\mu^{T}=\mu^{*}$, then $t(4 c-2 \delta \beta)\left(\mu^{*}-\mu_{i}\right)=\delta u_{T}$. Using (36), the gain from opt-out when $\mu^{T}=\mu^{*}$ is

$$
-\delta \mu^{*}\left(u_{T}+2 \mu^{*} \Delta u+\frac{3}{2} u_{T}\right)+u_{T}+2 \mu^{*} \Delta u-\frac{\delta u_{T}}{2} \mu_{i},
$$

which is less than

$$
-\frac{2}{3} u_{T}-\frac{4}{3} \mu^{*} \Delta u-u_{T}+u_{T}+2 \mu^{*} \Delta u-\frac{\delta u_{T}}{2} \mu_{i}=-\frac{2}{3}\left(u_{T}-\mu^{*} \Delta u\right)-\frac{\delta u_{T}}{2} \mu_{i}<0 .
$$

Therefore, the gain from opt-out is negative for all $\mu^{T} \geq \mu^{*}$. And since $\delta \mu^{T}>1$ implies $\mu^{T}>\mu^{*}$ opt-out is not beneficial if $\delta \mu^{T}>1$.

\subsection{Proof of Proposition 10}

Proof. Assume for the moment that newspaper 2 chooses $p_{2}=0$ and does not block the traffic from the aggregator. Then, the market share of newspaper 1 is

$$
\alpha_{1}=\frac{1}{2}-\frac{1}{t} \frac{\left(\mu\left(s_{2}\right)-\mu\left(s_{1}\right)\right) \Delta u+u_{T}+p_{1}}{1+\mu\left(s_{2}\right)}
$$

and its profit is given by

$$
\pi_{1}=\alpha_{1}\left(1+\delta \mu_{1}+p_{1}\right)-c \mu_{1}^{2}
$$

Since the profit function is concave with respect to price, it is sufficient to show $\left.\frac{\partial \pi_{1}}{\partial p_{1}}\right|_{p_{1}=0}<0$. We have

$$
\left.\frac{\partial \pi_{1}}{\partial p_{1}}\right|_{p_{1}=0}<0 \Leftrightarrow \frac{t}{2}\left(1+\mu_{2}\right)-\delta \mu_{1}-1+\left(\mu_{1}-\mu_{2}\right) \Delta u-u_{T}<0 .
$$


This is satisfied for any $\left(\mu_{1}, \mu_{2}\right) \in[0,1 / 2]^{2}$, if $t<4 / 3$. Since $t<4 / 3$ implies

$$
\frac{t}{2}\left(1+\mu_{2}\right)-1<0
$$

we have

$$
\frac{t}{2}\left(1+\mu_{2}\right)-\delta \mu_{1}-1+\left(\mu_{1}-\mu_{2}-1 / 2\right) \Delta u<0
$$

Hence, from A4 we can conclude $\left.\frac{\partial \pi_{1}}{\partial p_{1}}\right|_{p_{1}=0}<0$. This shows that if $t<4 / 3, p_{1}=0$ is a best response to $p_{2}=0$. In addition, our proof proves that $p_{1}=0$ is best response for $p_{2}>0$ since $p_{2}>0$ (and hence blocking the traffic from the aggregator) corresponds to the special case of $\mu_{2}=0$ and the proof works for this case.

\subsection{Proof of Proposition 11}

(i) When both newspapers charge prices, the market share of newspaper 1 is

$$
\alpha_{1}=\frac{1}{2}+\frac{\left(\mu_{1}-\mu_{2}\right) \Delta u+\left(p_{2}-p_{1}\right)}{2 t} .
$$

Newspaper's 1 profit is

$$
\pi_{1}=\alpha_{1}\left(1+\delta \mu_{1}+p_{1}\right)-c \mu_{1}^{2}
$$

Given $\mu_{1}$, and $\mu_{2}$, from the first-order condition with respect to $p_{1}$, we find 1's best response price as follows.

$$
B R_{1}\left(p_{1}, \mu_{1}, \mu_{2}\right)=\frac{t}{2}+\frac{\Delta u}{2}\left(\mu_{1}-\mu_{2}\right)+\frac{p_{2}}{2}-\frac{1}{2}-\frac{\delta \mu_{1}}{2} .
$$

$B R_{2}()$ is similarly obtained. Therefore, the equilibrium price of 1 for given qualities is

$$
p_{1}=t+\frac{\Delta u}{3}\left(\mu_{1}-\mu_{2}\right)-1-\frac{2 \delta \mu_{1}}{3}-\frac{\delta \mu_{2}}{3},
$$

implying

$$
\begin{gathered}
p_{2}-p_{1}=\frac{1}{3}\left(\mu_{2}-\mu_{1}\right)(2 \Delta u-\delta), \\
\alpha_{1}=\frac{1}{2}+\frac{\left.\left(\mu_{1}-\mu_{2}\right)(\Delta u+\delta)\right)}{6 t}, \\
\pi_{1}=\frac{1}{2 t}\left(t+\frac{1}{3}\left(\mu_{1}-\mu_{2}\right)(\Delta u+\delta)\right)^{2}-c \mu_{1}^{2}
\end{gathered}
$$


From the first order condition with respect to $\mu_{1}$, we obtain the equilibrium quality under paywall, $\mu^{P}$, in the symmetric equilibrium, $\mu_{1}=\mu_{2}$, as follows.

$$
\mu^{P}=\frac{\Delta u+\delta}{6 c}
$$

The equilibrium profit under paywall is

$$
\pi^{P}=\frac{t}{2}-c \mu^{P^{2}}
$$

(ii) We have

$$
\begin{aligned}
\mu^{P} & <\mu^{*} \Leftrightarrow \\
\frac{\Delta u+\delta}{6 c} & <\frac{\frac{\Delta u}{t}+\delta}{4 c-\delta \frac{\Delta u}{t}} \Leftrightarrow \\
4 c(\Delta u+\delta)-\delta \frac{\Delta u}{t}(\Delta u+\delta) & <6 c \frac{\Delta u}{t}+6 c \delta \Leftrightarrow \\
c\left(4 \Delta u-2 \delta-6 \frac{\Delta u}{t}\right) & <\delta \frac{\Delta u}{t}(\Delta u+\delta)
\end{aligned}
$$

Since the RHS is always positive, it is sufficient to show that the LHS is negative. We show $(4-6 / t) \Delta u<0$.

$$
t<\frac{4}{3} \Leftrightarrow-\frac{1}{t}<-\frac{3}{4} \Leftrightarrow 4-\frac{6}{t}<4-\frac{18}{4}=-\frac{1}{2}
$$

(iii)

$$
\begin{aligned}
\pi^{P} & >\pi^{*} \Leftrightarrow \\
\frac{t}{2}-c \mu^{P^{2}} & >\frac{1}{2}+\frac{\delta}{2} \mu^{*}-c \mu^{*^{2}} \Leftrightarrow \\
\left(\mu^{*}-\mu^{P}\right)\left(c \mu^{*}+c \mu^{P}\right)+\left(\frac{t}{2}-\frac{1}{2}-\frac{\delta}{2} \mu^{*}\right) & >0 \Leftrightarrow \\
\left(\mu^{*}-\mu^{P}\right)\left(c \mu^{*}+c \mu^{P}-\frac{\delta}{2}\right)+\left(\frac{t}{2}-\frac{1}{2}-\frac{\delta}{2} \mu^{P}\right) & >0 \Leftrightarrow
\end{aligned}
$$

We know $t-1-\delta \mu^{p}$ is the equilibrium price and therefore is positive. We also know $\mu^{*}>\mu^{P}$. Therefore, $\pi^{P}>\pi^{*}$, if $c \mu^{*}+c \mu^{P}-\frac{\delta}{2}>0$. We have:

$$
c \mu^{*}+c \mu^{P}-\frac{\delta}{2}>2 c \mu^{P}-\frac{\delta}{2}=\frac{\Delta u+\delta}{3}-\frac{\delta}{2}=\frac{2 \Delta u-\delta}{6}>0 .
$$

